\title{
A positivity preserving property result for the biharmonic operator under partially hinged boundary conditions
}

\author{
Elvise Berchio ${ }^{1} \cdot$ Alessio Falocchi $^{1}$ (D)
}

Received: 19 August 2020 / Accepted: 4 November 2020 / Published online: 27 November 2020

(c) The Author(s) 2020

\begin{abstract}
It is well known that for higher order elliptic equations, the positivity preserving property (PPP) may fail. In striking contrast to what happens under Dirichlet boundary conditions, we prove that the PPP holds for the biharmonic operator on rectangular domains under partially hinged boundary conditions, i.e., nonnegative loads yield positive solutions. The result follows by fine estimates of the Fourier expansion of the corresponding Green function.
\end{abstract}

Keywords Biharmonic $\cdot$ Positivity preserving $\cdot$ Partially hinged plate $\cdot$ Green function

Mathematics Subject Classification $35 \mathrm{G} 15 \cdot 35 \mathrm{~J} 08 \cdot 35 \mathrm{~B} 09$

\section{Introduction}

One of the main obstructions in the development of the theory of higher order elliptic equations is represented by the loss of general maximum principles, see, e.g., [9, Chapter 1]. Nevertheless, due to the central role that these technical tolls play in the general theory of second-order elliptic equations, in the last century a large part of literature has focused in studying whether the related boundary-value problems possibly enjoy the so-called positivity preserving property (PPP in the following). As a matter of example, let us consider the clamped plate problem:

$$
\begin{cases}\Delta^{2} u=f & \text { in } \Omega \\ u=|\nabla u|=0 & \text { on } \partial \Omega\end{cases}
$$

where $\Omega \subset \mathbb{R}^{n}$ is a bounded domain and $f \in L^{2}(\Omega)$; we say that the above problem satisfies the PPP if the following implication holds

$$
f \geqslant 0 \text { in } \Omega \quad \Rightarrow \quad u \geqslant 0 \text { in } \Omega,
$$

Alessio Falocchi

elvise.berchio@polito.it

1 Dipartimento di Scienze Matematiche, Politecnico di Torino, Corso Duca degli Abruzzi 24, 10129 Turin, Italy 
where $u$ is a (weak) solution to (1). The validity of the PPP generally depends either on the choice of the boundary conditions or on the geometry of the domain. For instance, from the seminal works by Boggio [5, 6], it is known that problem (1) satisfies the PPP when $\Omega$ is a ball in $\mathbb{R}^{n}$, while, in [7], Coffman and Duffin proved that the PPP does not hold when $\Omega$ is a two-dimensional domain containing a right angle, such as a square or a rectangle.

Things become somehow simpler if in (1), instead of the Dirichlet boundary conditions, we take the Navier boundary conditions, i.e., we consider the hinged plate problem:

$$
\begin{cases}\Delta^{2} u=f & \text { in } \Omega \\ u=\Delta u=0 & \text { on } \partial \Omega .\end{cases}
$$

Here, the PPP follows by applying twice the comparison principle for the Laplacian under Dirichlet boundary conditions. It is worth noticing that smoothness of the domain cannot be overlooked since it has been shown by Nazarov and Sweers [15] that, also in this case, the PPP may fail for planar domains with an interior corner. We refer to the book [9] for more details and PPP results under different kinds of boundary conditions, e.g., Steklov boundary conditions, and to [10-12, 16-19, 21] for up-to-date results on the topic.

In the present paper, we focus on the less studied partially hinged plate problem which arises in several mathematical models having engineering interest, e.g., models of bridges or footbridges. In particular, a 2-d model for suspension bridges has been proposed in [8]; here, the bridge is seen as a thin long rectangular plate $\Omega \subset \mathbb{R}^{2}$ hinged at the short edges, see also [4] for further details. More precisely, if, by scaling, we assume that $\Omega=(0, \pi) \times(-d, d)$ with $d>0$, the partially hinged problem writes:

$$
\begin{cases}\Delta^{2} u=f & \text { in } \Omega \\ u(0, y)=u_{x x}(0, y)=u(\pi, y)=u_{x x}(\pi, y)=0 & \text { for } y \in(-d, d) \\ u_{y y}(x, \pm d)+\sigma u_{x x}(x, \pm d)=u_{y y y}(x, \pm d)+(2-\sigma) u_{x x y}(x, \pm d)=0 & \text { for } x \in(0, \pi)\end{cases}
$$

where $f \in L^{2}(\Omega), \sigma \in[0,1)$ is the so-called Poisson ratio and depends on the material by which the plate is made of. It is known that the validity of the PPP for a problem is related to the sign of the associated Green function. Indeed, if $G_{p}(q):=G(p, q)$ denotes the Green function of (2), the (weak) solution to (2) writes

$$
u(q)=\int_{\Omega} G_{p}(q) f(p) d p \quad \forall q \in \Omega
$$

and the PPP becomes equivalent to

$$
G_{p}(q) \geqslant 0 \quad \forall(p, q) \in \Omega .
$$

The proof of the above inequality represents the main result of the present paper. More precisely, we first write the Fourier expansion of $G_{p}$, i.e.,

$$
G_{p}(q)=\sum_{m=1}^{+\infty} \frac{1}{2 \pi} \frac{\phi_{m}(y, \eta)}{m^{3}} \sin (m \xi) \sin (m x) \quad \forall p=(\xi, \eta) \text { and } q=(x, y) \in \bar{\Omega},
$$

where the (involved) analytic expression of the functions $\phi_{m}$ is given explicitly in formula (15) of Sect. 3. We remark that, differently from what it happens in the second-order case, the use of a single Fourier series solution is not common for fourth-order problems; indeed, it often does not work; for plates with two opposite edges simply supported, the 
effectiveness of its use was highlighted by Lévy in 1899 and we refer to [14, Section 2.2] for a discussion of the original Lévy method and its generalizations. Nevertheless, boundary conditions (2) were not considered in [14] and they require some additional effort, see, e.g., [8] and Sect. 3.

As a subsequent step, in the article we develop an accurate analysis of the qualitative properties of the $\phi_{m}$ and we show, in particular, that they are strictly decreasing with respect to $m \in \mathbb{N}^{+}$. This monotonicity issue is achieved by studying the sign of the derivatives of the $\phi_{m}$; since they have highly involved analytic expressions, in order to detect their sign, we set up a clever scheme where, step by step, we cancel out the dependence of some variables through optimization arguments, see Remark 4.1 of Sect. 4. From the monotonicity of the $\phi_{m}$, through an asymptotic analysis, we also deduce their positivity. These pieces of information are essential for the subsequent part of the proof where we study the sign of $G_{p}$. More precisely, by means of suitable lower bounds, we first show the positivity of $G_{p}$ in a rectangle contained in $\Omega$, far from the hinged edges; then, we obtain the positivity in the remaining parts through suitable iterative procedures which, step by step, stick rectangles where $G_{p}$ is positive up to the boundary, see Sect. 5 for all details.

As already remarked, the validity of the PPP for problem (2) is by no means an obvious fact; recall that it does not hold for problem (1) on rectangular planar domains; by numerical observations, we do not even expect its validity for the partially clamped plate problem, i.e., (2) with Dirichlet conditions instead of Navier, see Figure 1 on the right. Furthermore, we believe that the validity of the PPP will help in making a significant step forward in the spectral analysis of the operator in (2) and in the related stability analysis for partially hinged plates, especially in the nonhomogeneous case, see, e.g., [2] and [3].

The paper is organized as follows. In Sect. 2, we introduce some notations and we state our main results: the Fourier expansion of $G_{p}$, together with the qualitative properties of its components, which is given in Theorem 2.1 and the precise statement of the PPP result which is given in Theorem 2.2. The rest of the paper is devoted to the proofs. More precisely, in Sect. 3 we compute explicitly the Fourier series of the Green function as the limit of the solution to (2) for a specific $L^{2}$ forcing term converging to the Dirac delta function. In Sect. 4 we prove the monotonicity and the positivity of the $\phi_{m}$, while in Sect. 5 we show the positivity of the Green function. Finally, we collect in "Appendix" the proofs of some technical results needed either in Sect. 4 or in Sect. 5 .

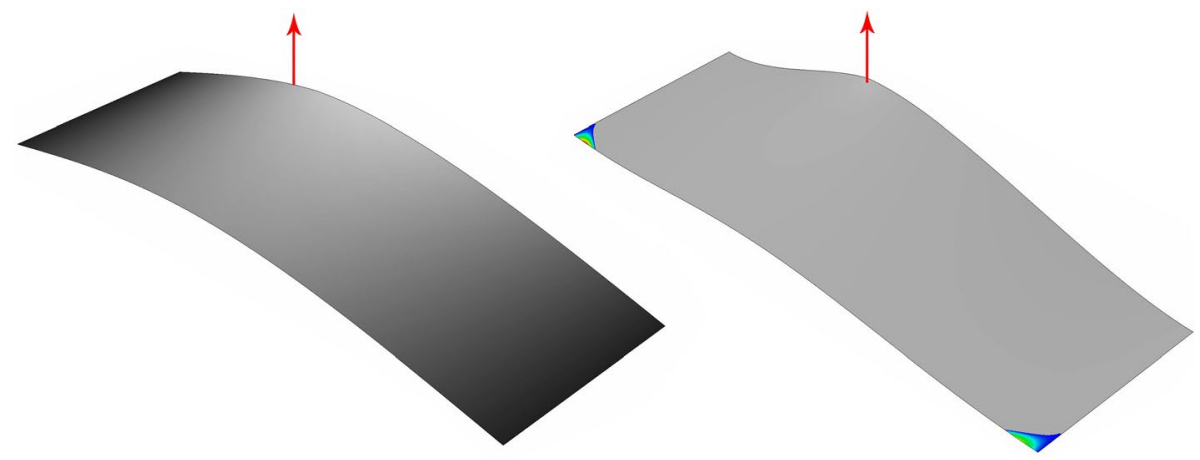

Fig. 1 Finite element approximated solution $u$ of the partially hinged (left) and partially clamped (right) plate problems under, respectively, a concentrated load in $\left(\frac{\pi}{3}, d\right)$; the regions where $u \geqslant 0$ are gray, while the regions where $u<0$ are colored from blue (less negative) to red ( $d=\pi / 6, \sigma=0.2$ ) 


\section{Notations and main results}

The natural functional space where to set problem (2) is

$$
H_{*}^{2}(\Omega)=\left\{u \in H^{2}(\Omega): u=0 \text { on }\{0, \pi\} \times(-d, d)\right\} .
$$

Note that the condition $u=0$ has to be meant in a classical sense because $\Omega$ is a planar domain and $H_{*}^{2}(\Omega) \subset C^{0}(\bar{\Omega})$. Furthermore, for $\sigma \in[0,1)$ fixed, by repeating the proof of [8, Lemma 4.1] with minor changes, $H_{*}^{2}(\Omega)$ is a Hilbert space when endowed with the scalar product

$$
(u, v)_{H_{*}^{2}(\Omega)}:=\int_{\Omega}\left[\Delta u \Delta v+(1-\sigma)\left(2 u_{x y} v_{x y}-u_{x x} v_{y y}-u_{y y} v_{x x}\right)\right] d x d y
$$

with associated norm $\|u\|_{H_{*}^{2}(\Omega)}^{2}=(u, u)_{H_{*}^{2}(\Omega)}$, which is equivalent to the usual norm in $H^{2}(\Omega)$. Then, we reformulate problem (2)in the following weak sense

$$
(u, v)_{H_{*}^{2}(\Omega)}=(f, v)_{L^{2}(\Omega)} \quad \forall v \in H_{*}^{2}(\Omega) .
$$

If $f \in H_{*}^{-2}(\Omega):=\left(H_{*}^{2}(\Omega)\right)^{\prime}$, we write $\langle f, v\rangle$ instead of $(f, v)_{L^{2}(\Omega)}$, i.e.,

$$
(u, v)_{H_{*}^{2}(\Omega)}=\langle f, v\rangle \quad \forall v \in H_{*}^{2}(\Omega) .
$$

Clearly, problem (4) (and consequently (3)) admits a unique solution $u \in H_{*}^{2}(\Omega)$; in the following, we shall specify the cases when $f \in H_{*}^{-2}(\Omega)$, otherwise we will always assume $f \in L^{2}(\Omega)$. For all $p \in \Omega$, the Green function $G_{p}$ of (2) is, by definition, the unique solution to

$$
\left(G_{p}, v\right)_{H_{*}^{2}(\Omega)}=\left\langle\delta_{p}, v\right\rangle=v(p) \quad \forall v \in H_{*}^{2}(\Omega) .
$$

By separating variables, in Sect. 3 we derive the Fourier expansion of $G_{p}$ and in Sect. 4 we prove some crucial qualitative properties of its Fourier components. We collect these results in the following:

Theorem 2.1 Let $\sigma \in[0,1)$ and $p=(\xi, \eta) \in \bar{\Omega}$, and furthermore let $G_{p} \in H_{*}^{2}(\Omega)$ be the Green function of (2). Then,

$$
G_{p}(x, y)=\frac{1}{2 \pi} \sum_{m=1}^{+\infty} \frac{\phi_{m}(y, \eta)}{m^{3}} \sin (m \xi) \sin (m x) \quad \forall(x, y) \in \bar{\Omega},
$$

where the functions $\phi_{m}(y, \eta)$ are given explicitly in formula (15) of Sect. 3. In particular, the $\phi_{m}(y, \eta)$ are strictly positive and strictly decreasing with respect to $m$, i.e.,

$$
0<\phi_{m+1}(y, \eta)<\phi_{m}(y, \eta) \quad \forall m \in \mathbb{N}^{+}, \forall y, \eta \in[-\ell, \ell] .
$$

In Figure 2 on the left, we provide the plot of $\phi_{1}(y, \eta)$ with $d=\pi / 150$ and $\sigma=0.2$; on the right, we provide the plot of $\phi_{1}(y, \eta)$ and $\phi_{2}(y, \eta)$ for $d=3 \pi / 4$. Qualitatively, we have similar plots for any $m \in \mathbb{N}^{+}$and they all highlight that the points where the positivity of $\phi_{m}(y, \eta)$ is more difficult to show are $( \pm d, \mp d)$. This confirms the physical intuition that a concentrated load in $w=d$ produces the largest vertical (positive) displacement in $y=d$ and the smallest 

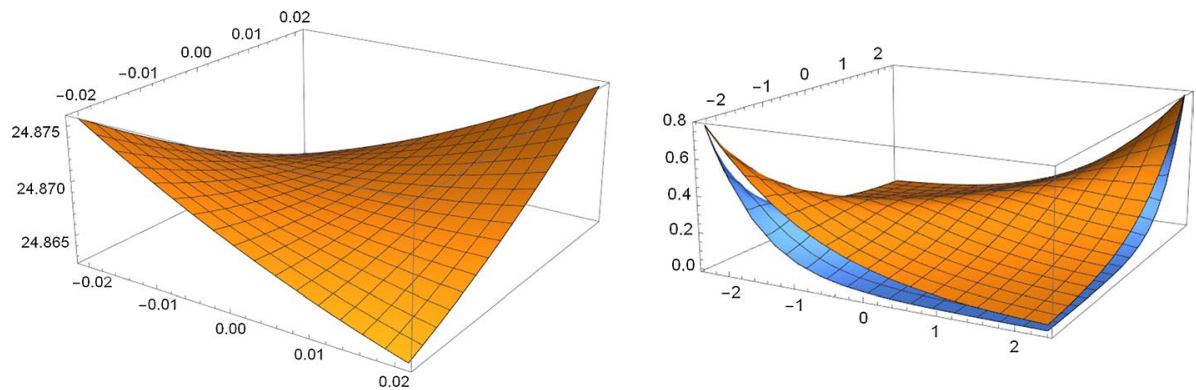

Fig. 2 On the left plot of $\phi_{1}(y, \eta)$ with $d=\pi / 150$ and $\sigma=0.2$; on the right plot of $\phi_{1}(y, \eta)$ (orange) and $\phi_{2}(y, \eta)$ (blue) with $d=3 \pi / 4$ and $\sigma=0.2$

in $y=-d$. We refer to [1] for a detailed analysis about the torsional performances of partially hinged plates under the action of different external forces. Instead, Figure 2 on the right highlights how the monotonicity issue (with respect $m$ ) becomes more difficult to be proved at $( \pm d, \pm d)$, where the difference between the $\phi_{m}$ reduces. Numerically, we see that this becomes more evident for large $d$. However, Theorem 2.1 assures that the $\phi_{m}$ never intersect and preserve their positivity for all $d>0$.

By exploiting Theorem 2.1, we derive the main result of the paper, namely the positivity of $G_{p}$. More precisely, we set

$$
\widetilde{\Omega}:=(0, \pi) \times[-d, d]
$$

and we prove

Theorem 2.2 Let $\sigma \in[0,1)$ and $p \in \widetilde{\Omega}$, and furthermore let $G_{p}$ be the Green function of (2). There holds

$$
G_{p}(x, y)>0 \quad \forall(x, y) \in \widetilde{\Omega} .
$$

Therefore, if $f \in L^{2}(\Omega)$ and $u$ is the solution of (2), the following implication holds

$$
f \geqslant 0, f \not \equiv 0 \text { in } \Omega \Rightarrow u>0 \text { in } \widetilde{\Omega} .
$$

Remark 2.3 The Poisson ratio $\sigma$ of a material is defined as the ratio between the transversal strain and the longitudinal strain in the direction of the stretching force; for most of materials, we have $\sigma \in(0,1 / 2)$. Nevertheless, there are materials having negative Poisson ratio; hence, the range $\sigma \in(-1,1 / 2)$ includes all possible values. Numerical experiments lead us to conjecture that Theorem 2.2 still holds for $\sigma \in(-1,0)$. In Remark 4.2 of Sect. 4, we highlight the points where our proof fails when assuming $\sigma$ negative. 


\section{Green function computation}

The aim of this section is to provide the Fourier expansion of the Green function $G_{p}$, namely of the solution to (5). This is done by developing a suitable limit approach where, in principle, $\delta_{p}$ is replaced by a suitable $L^{2}$ function converging to it. To begin with, we fix $p=(\xi, \eta) \in \Omega$ and we introduce $\alpha, \beta>0$ sufficiently small so that $[\xi-\alpha, \xi+\alpha] \times[\eta-\beta, \eta+\beta] \subset \Omega$; then, we denote by $u_{\alpha, \beta}^{p} \in H_{*}^{2}(\Omega)$ the unique solution to the auxiliary problem:

$$
\left(u_{\alpha, \beta}^{p}, v\right)_{H_{*}^{2}(\Omega)}=\left(f_{\alpha, \beta}^{p}, v\right)_{L^{2}(\Omega)} \quad \forall v \in H_{*}^{2}(\Omega),
$$

where

$$
f_{\alpha, \beta}^{p}(x, y):=\frac{\chi_{[\xi-\alpha, \xi+\alpha]}(x) \chi_{[\eta-\beta, \eta+\beta]}(y)}{4 \alpha \beta}
$$

and $\chi_{A}$ denotes the characteristic function of the set $A \subset \mathbb{R}$. Recalling that $H_{*}^{2}(\Omega) \subset C^{0, \gamma}(\bar{\Omega})$ for any $0<\gamma<1$, by the mean value theorem there exists $\left(\sigma_{\alpha, \beta}, \tau_{\alpha, \beta}\right) \in[\xi-\alpha, \xi+\alpha] \times[\eta-\beta, \eta+\beta]$ such that

$$
\left|\left\langle f_{\alpha, \beta}^{p}-\delta_{p}, v\right\rangle\right|=\left|v\left(\sigma_{\alpha, \beta}, \tau_{\alpha, \beta}\right)-v(\xi, \eta)\right| \leqslant C\left|\left(\sigma_{\alpha, \beta}, \tau_{\alpha, \beta}\right)-(\xi, \eta)\right|^{\gamma}\|v\|_{H_{*}^{2}(\Omega)},
$$

for all $v \in H_{*}^{2}(\Omega)$ and for some $C>0$. Since $\left(\sigma_{\alpha, \beta}, \tau_{\alpha, \beta}\right) \rightarrow(\xi, \eta)$ as $(\alpha, \beta) \rightarrow(0,0)$, we infer that $f_{\alpha, \beta}^{p} \rightarrow \delta_{p}$ in $H_{*}^{-2}(\Omega)$ and, in turn, that

$$
u_{\alpha, \beta}^{p} \rightarrow G_{p} \quad \text { in } H_{*}^{2}(\Omega) \quad \text { as }(\alpha, \beta) \rightarrow(0,0) .
$$

Next, we provide the explicit Fourier expansion of $u_{\alpha, \beta}^{p}$. To this aim, we set:

$$
\begin{aligned}
c_{1}:= & c_{1}(m, \eta, \beta)= \\
& \frac{m A(m d)\left[V_{m, \eta, \beta}(d)+V_{m, \eta, \beta}(-d)\right]+B(m d)\left[W_{m, \eta, \beta}(d)-W_{m, \eta, \beta}(-d)\right]}{2 m^{3}(1-\sigma) F(m d)} \\
c_{2}:= & c_{2}(m, \eta, \beta)= \\
& \frac{m \bar{A}(m d)\left[V_{m, \eta, \beta}(d)-V_{m, \eta, \beta}(-d)\right]+\bar{B}(m d)\left[W_{m, \eta, \beta}(d)+W_{m, \eta, \beta}(-d)\right]}{2 m^{3}(1-\sigma) \bar{F}(m d)} \\
c_{3}:= & c_{3}(m, \eta, \beta)= \\
& \frac{m \cosh (m d)\left[V_{m, \eta, \beta}(d)-V_{m, \eta, \beta}(-d)\right]-\sinh (m d)\left[W_{m, \eta, \beta}(d)+W_{m, \eta, \beta}(-d)\right]}{2 m^{2} \bar{F}(m d)} \\
c_{4}:= & c_{4}(m, \eta, \beta)= \\
& \frac{m \sinh (m d)\left[V_{m, \eta, \beta}(d)+V_{m, \eta, \beta}(-d)\right]-\cosh (m d)\left[W_{m, \eta, \beta}(d)-W_{m, \eta, \beta}(-d)\right]}{2 m^{2} F(m d)}
\end{aligned}
$$

where the functions $F, \bar{F}, A, \bar{A}, B, \bar{B}:(0,+\infty) \rightarrow \mathbb{R}$ and $V_{m, \eta, \beta}, W_{m, \eta, \beta}, \Phi_{m, \eta, \beta}:[-d, d] \rightarrow \mathbb{R}$ are defined as follows

$$
F(z):=\frac{(3+\sigma)}{2} \sinh (2 z)-z(1-\sigma), \quad \bar{F}(z):=\frac{(3+\sigma)}{2} \sinh (2 z)+z(1-\sigma),
$$




$$
\begin{aligned}
& A(z):=(1+\sigma) \sinh (z)-(1-\sigma) z \cosh (z), \\
& \bar{A}(z):=(1+\sigma) \cosh (z)-(1-\sigma) z \sinh (z), \\
& B(z):=2 \cosh (z)+(1-\sigma) z \sinh (z), \\
& \bar{B}(z):=2 \sinh (z)+(1-\sigma) z \cosh (z) \\
& V_{m, \eta, \beta}(y):=\sigma m^{2} \Phi_{m, \eta, \beta}(y)-\left(\Phi_{m, \eta, \beta}\right)^{\prime \prime}(y), \\
& W_{m, \eta, \beta}(y):=(\sigma-2) m^{2}\left(\Phi_{m, \eta, \beta}\right)^{\prime}(y)+\left(\Phi_{m, \eta, \beta}\right)^{\prime \prime \prime}(y),
\end{aligned}
$$

with

$$
\Phi_{m, \eta, \beta}(y):=\frac{1}{2 \beta} \int_{\eta-\beta}^{\eta+\beta} \frac{(1+m|y-t|) e^{-m|y-t|}}{4 m^{3}} d t .
$$

$\Phi_{m, \eta, \beta}$ is given by the convolution of the $H^{3}(\mathbb{R})$ function $\frac{(1+m|y|) e^{-m|y|}}{4 m^{3}}$ and the $L^{2}(\mathbb{R})$ function $\frac{\chi_{[\eta-\beta, \eta+\beta]}(y)}{2 \beta}$, hence $\Phi_{m, \eta, \beta} \in C^{3}(\mathbb{R})$, and all the above constants are well defined.

We prove

Lemma 3.1 Let $u_{\alpha, \beta}^{p}$ be the unique solution to (7), then

$$
u_{\alpha, \beta}^{p}(x, y)=\sum_{m=1}^{+\infty} \varphi_{m, \alpha, \beta}^{p}(y) \sin (m x)
$$

with

$$
\begin{gathered}
\varphi_{m, \alpha, \beta}^{p}(y):=\frac{2}{\pi} \frac{\sin (m \alpha)}{m \alpha} \sin (m \xi)\left[c_{1} \cosh (m y)+c_{2} \sinh (m y)+c_{3} y \cosh (m y)\right. \\
\left.+c_{4} y \sinh (m y)+\Phi_{m, \eta, \beta}(y)\right]
\end{gathered}
$$

where the constants $c_{i}$ and $\Phi_{m, \underline{\eta}, \beta}$ are defined in (9) and (11). Furthermore, the above series converges in $H_{*}^{2}(\Omega)$ and in $C^{0}(\Omega)$.

Proof First, we denote the Fourier coefficients of $f_{\alpha, \beta}^{p}$ by

$$
f_{m, \alpha, \beta}^{p}(y):=\frac{2}{\pi} \int_{0}^{\pi} f_{\alpha, \beta}^{p}(x, y) \sin (m x) d x=\frac{\chi_{[\eta-\beta, \eta+\beta]}(y)}{\pi \beta} \frac{\sin (m \alpha)}{m \alpha} \sin (m \xi) .
$$

Then, for $M \geqslant 1$ we define

$$
u_{\alpha, \beta}^{p, M}(x, y)=\sum_{m=1}^{M} \varphi_{m, \alpha, \beta}^{p}(y) \sin (m x)
$$

where, for each $1 \leqslant m \leqslant M, \varphi_{m}=\varphi_{m, \alpha, \beta}^{p}(y)$ is the unique solution to the problem:

$$
a_{m}\left(\varphi_{m}, \phi\right)=\left(f_{m, \alpha, \beta}^{p}, \phi\right)_{L^{2}(-d, d)} \quad \forall \phi \in H^{2}(-d, d),
$$

with 


$$
a_{m}(\varphi, \phi):=\int_{-d}^{d}\left[\varphi^{\prime \prime} \phi^{\prime \prime}+2 m^{2}(1-\sigma) \varphi^{\prime} \phi^{\prime}-\sigma m^{2}\left(\varphi^{\prime \prime} \phi+\varphi \phi^{\prime \prime}\right)+m^{4} \varphi \phi\right] d y
$$

continuous and coercive bilinear form in $H^{2}(-d, d)$ with associated norm $\|\varphi\|_{H_{m}^{2}(-d, d)}^{2}:=a_{m}(\varphi, \varphi)$. In strong form, problem (13) reads

$$
\left\{\begin{array}{l}
\varphi_{m}^{\prime \prime \prime \prime}(y)-2 m^{2} \varphi_{m}^{\prime \prime}(y)+m^{4} \varphi_{m}(y)=f_{m, \alpha, \beta}^{p}(y) y \in(-d, d) \\
\varphi_{m}^{\prime \prime}( \pm d)-\sigma m^{2} \varphi_{m}( \pm d)=0 \\
\varphi_{m}^{\prime \prime \prime}( \pm d)-(2-\sigma) m^{2} \varphi_{m}^{\prime}( \pm d)=0
\end{array}\right.
$$

and some computations yield that the $\varphi_{m, \alpha, \beta}^{p}$ are as in (12). Let $v \in H_{*}^{2}(\Omega)$, it is readily checked that, for $M \geqslant 1$ fixed, $u_{\alpha, \beta}^{p, M}$ satisfies

$$
\left(u_{\alpha, \beta}^{p, M}, v^{M}\right)_{H_{*}^{2}(\Omega)}=\left(f_{\alpha, \beta}^{p, M}, v^{M}\right)_{L^{2}(\Omega)},
$$

where $v^{M}$ and $f_{\alpha, \beta}^{p, M}$ denote, respectively, the partial sums of the Fourier expansion of $v$ and $f_{\alpha, \beta}^{p}$. By the standard theory of Fourier series, we get $f_{\alpha, \beta}^{p, M} \rightarrow f_{\alpha, \beta}^{p}$ in $L^{2}(\Omega)$ and $v^{M} \rightarrow v$ in $H_{*}^{2}(\Omega)$ as $M \rightarrow+\infty$. Furthermore, since

$$
\left\|u_{\alpha, \beta}^{p, M}\right\|_{H_{*}^{2}(\Omega)}^{2}=\frac{\pi}{2} \sum_{m=1}^{M}\left\|\varphi_{m, \alpha, \beta}^{p}\right\|_{H_{m}^{2}(-d, d)}^{2}=\frac{\pi}{2} \sum_{m=1}^{M}\left\|f_{m, \alpha, \beta}^{p}\right\|_{L^{2}(-d, d)}^{2} \leqslant\left\|f_{\alpha, \beta}^{p}\right\|_{L^{2}(\Omega)}^{2}
$$

we have that $u_{\alpha, \beta}^{p, M} \rightarrow u_{\alpha, \beta}^{p}$ in $H_{*}^{2}(\Omega)$ as $M \rightarrow+\infty$. Finally, the proof of Lemma 3.1 follows by passing to the limit in (14).

In order to write explicitly the Fourier expansion of $G_{p}$, for all $m \in \mathbb{N}_{+}$, we set

$$
\begin{aligned}
\phi_{m}(y, \eta):=e^{-m d}\left[\cosh (m \eta)\left(\frac{\bar{\zeta}(m y, m d)}{F(m d)}+m d \frac{\bar{\psi}(m y, m d)}{F(m d)}-m \eta \frac{\bar{\omega}(m y, m d)}{\bar{F}(m d)}\right)\right. \\
\left.\quad+\sinh (m \eta)\left(\frac{\bar{\vartheta}(m y, m d)}{\bar{F}(m d)}+m d \frac{\bar{\omega}(m y, m d)}{\bar{F}(m d)}-m \eta \frac{\bar{\psi}(m y, m d)}{F(m d)}\right)\right] \\
+(1+m|y-\eta|) e^{-m|y-\eta|}
\end{aligned}
$$

with the functions $F, \bar{F}$ as in $(10)$ and $\bar{\zeta}, \bar{\vartheta}, \bar{\psi}, \bar{\omega}: \mathbb{R} \times(0,+\infty) \rightarrow \mathbb{R}$ defined as follows

$$
\begin{aligned}
\bar{\zeta}(r, z):= & \left(\frac{4}{1-\sigma}-z(1+\sigma)\right) \cosh (r) \cosh (z)+\left(\frac{(1+\sigma)^{2}}{1-\sigma}+2 z\right) \cosh (r) \sinh (z) \\
& -2 r \sinh (r) \cosh (z)+r(1+\sigma) \sinh (r) \sinh (z) \\
\bar{\vartheta}(r, z):= & r(1+\sigma) \cosh (r) \cosh (z)-2 r \cosh (r) \sinh (z) \\
& +\left(\frac{(1+\sigma)^{2}}{1-\sigma}+2 z\right) \sinh (r) \cosh (z)+\left(\frac{4}{1-\sigma}-z(1+\sigma)\right) \sinh (r) \sinh (z) \\
\bar{\psi}(r, z):= & (2+(1-\sigma) z) \cosh (r) \cosh (z)+(-(1+\sigma)+z(1-\sigma)) \cosh (r) \sinh (z) \\
& -r(1-\sigma) \sinh (r) \cosh (z)-r(1-\sigma) \sinh (r) \sinh (z) \\
\bar{\omega}(r, z):= & -r(1-\sigma) \cosh (r) \cosh (z)-r(1-\sigma) \cosh (r) \sinh (z) \\
& +(-(1+\sigma)+z(1-\sigma)) \sinh (r) \cosh (z)+(2+(1-\sigma) z) \sinh (r) \sinh (z) .
\end{aligned}
$$


From Lemma 3.1, we get:

Proposition 3.2 Let $\sigma \in[0,1)$ and $p=(\xi, \eta) \in \bar{\Omega}$, and furthermore let $G_{p} \in H_{*}^{2}(\Omega)$ be as in (5). Then,

$$
G_{p}(x, y)=\frac{1}{2 \pi} \sum_{m=1}^{+\infty} \frac{\phi_{m}(y, \eta)}{m^{3}} \sin (m \xi) \sin (m x) \quad \forall(x, y) \in \bar{\Omega},
$$

where the functions $\phi_{m}(y, \eta)$ are given in (15).

Proof By expanding in Fourier series, we have: $G_{p}(x, y)=\sum_{m=1}^{+\infty} g_{m}^{p}(y) \sin (m x)$. Let $u_{\alpha, \beta}^{p} \in H_{*}^{2}(\Omega)$ be the unique solution to (7), by Lemma 3.1 its Fourier coefficients $\varphi_{m, \alpha, \beta}^{p}(y)$ write as in (12) and, by (8), $\varphi_{m, \alpha, \beta}^{p}(y) \rightarrow g_{m}^{p}(y)$ in $C^{0}([-d, d])$ as $(\alpha, \beta) \rightarrow(0,0)$.

Since, as $\beta \rightarrow 0$,

$$
\begin{aligned}
& \Phi_{m, \eta, \beta}(y) \rightarrow \frac{(1+m|y-\eta|) e^{-m|y-\eta|}}{4 m^{3}}:=\bar{\Phi}_{m}(y, \eta) \quad \text { in } C^{0}([-d, d]) \\
& V_{m, \eta, \beta}( \pm d) \rightarrow \frac{e^{-m| \pm d-\eta|}}{4 m}(1+\sigma-m| \pm d-\eta|(1-\sigma)), \\
& W_{m, \eta, \beta}( \pm d) \rightarrow \pm \frac{e^{-m| \pm d-\eta|}}{4}(2+m| \pm d-\eta|(1-\sigma)),
\end{aligned}
$$

from (12) we get

$$
\varphi_{m, \alpha, \beta}^{p}(y) \rightarrow \frac{\phi_{m}(y, \eta)}{2 \pi m^{3}} \sin (m \xi) \quad \text { as }(\alpha, \beta) \rightarrow(0,0),
$$

where the $\phi_{m}$ are as given in (15). This proves (17) for all $p \in \Omega$. Let now $\bar{p} \in \bar{\Omega}$ and let $G_{\bar{p}}$ be the corresponding solution to (5). It is readily seen that $\delta_{p_{n}} \rightarrow \delta_{\bar{p}}$ in $H_{*}^{-2}(\Omega)$ for all $\left\{p_{n}\right\} \subset \Omega: p_{n} \rightarrow \bar{p}$; then, arguing as in (8), it follows that $G_{p_{n}} \rightarrow G_{\bar{p}}$ in $H_{*}^{2}(\Omega)$ and, consequently, in $C^{0}(\bar{\Omega})$. By this, we infer that (17) extends continuously to all $p \in \bar{\Omega}$. The convergence of the series $(17)$ in $H_{*}^{2}(\Omega)$ and in $C^{0}(\bar{\Omega})$ can be easily checked by exploiting the monotonicity property (6) (see Sect. 4.1 for the proof). Indeed, we have

$$
\left|g_{m}^{p}(y) \sin (m x)\right| \leqslant \frac{1}{2 \pi} \frac{\phi_{m}(y, \eta)}{m^{3}}|\sin (m \xi)| \leqslant \frac{1}{2 \pi} \frac{\left\|\phi_{1}\right\|_{\infty}}{m^{3}} \leqslant \frac{C}{m^{3}},
$$

by which the convergence in $C^{0}(\bar{\Omega})$ follows. The convergence in $H_{*}^{2}(\Omega)$ comes from similar estimates.

Remark 3.3 An alternative and more neat way of writing the Green function expansion (17) is by means of the oscillating modes of the partially hinged plate $\Omega$; indeed, they have been explicitly computed in [8, Theorem 7.6] and have the form $\left\{\sin (m x) \Psi_{k, m}(y)\right\}_{k, m=1}^{\infty}$. However, the corresponding frequencies, which necessarily enter into this expansion, are only known implicitly by means of very involved equations. Therefore, in order to perform the explicit computations of the subsequent sections, it seems more convenient to follow the direct approach outlined in this section and write the Green function as in (17); clearly, the two-form solutions can be properly related. 


\section{Proof of Theorem 2.1}

The first part of the statement, namely the Fourier expansion of the Green function, has already been derived in the previous section, see Proposition 3.2. Here, we focus on the sign and monotonicity properties of the functions $\phi_{m}(y, \eta)$.

\subsection{Proof of the monotonicity issue in (6)}

We rewrite the $\phi_{m}(y, \eta)$ in a more convenient way; to this aim, we introduce the functions $\zeta, \vartheta, \psi, \omega:[-1,1] \times(0,+\infty) \rightarrow \mathbb{R}$

$$
\zeta(k, z):=\bar{\zeta}(k z, z), \vartheta(k, z):=\bar{\vartheta}(k z, z), \psi(k, z):=\bar{\psi}(k z, z), \omega(k, z):=\bar{\omega}(k z, z),
$$

where $\bar{\zeta}, \bar{\vartheta}, \bar{\psi}, \bar{\omega}$ are given in (16). We observe that

$$
\begin{array}{lcrl}
\zeta(-k, z)=\zeta(k, z) & \psi(-k, z)=\psi(k, z) & \forall k \in[-1,1] \forall z>0 \\
\vartheta(-k, z)=-\vartheta(k, z) & \omega(-k, z)=-\omega(k, z) & \forall k \in[-1,1] \forall z>0
\end{array}
$$

and the same symmetry properties hold for the derivatives with respect to $z$ of the previous functions.

Putting into (15) $z=m d>0, y=k d$ with $k \in[-1,1]$ and $\eta=s d$ with $s \in[-1,1]$, each $\phi_{m}(y, \eta)$ rewrites as the three-variable function:

$$
\phi(s, k, z)=e^{-z} g(s, k, z)+h(s, k, z),
$$

where

$$
\begin{aligned}
g(s, k, z):= & \cosh (s z)\left(\frac{\zeta(k, z)}{F(z)}+z \frac{\psi(k, z)}{F(z)}-s z \frac{\omega(k, z)}{\bar{F}(z)}\right) \\
& +\sinh (s z)\left(\frac{\vartheta(k, z)}{\bar{F}(z)}+z \frac{\omega(k, z)}{\bar{F}(z)}-s z \frac{\psi(k, z)}{F(z)}\right)
\end{aligned}
$$

and $h(s, k, z):=(1+z|k-s|) e^{-z|k-s|}$. The monotonicity issue (6) follows by showing that the function $\phi(s, k, z)$ is decreasing with respect to $z>0$ for all $k, s \in[-1,1]$, i.e.,

$$
\phi_{z}(s, k, z)=e^{-z}\left(g_{z}(s, k, z)-g(s, k, z)\right)+h_{z}(s, k, z)<0 \quad \forall z>0, \forall k, s \in[-1,1] .
$$

Since $h_{z}(s, k, z)=-(k-s)^{2} z e^{-z|k-s|} \leqslant 0$, for all $z>0$ and $k, s \in[-1,1]$, a sufficient condition for the validity of the above inequality is:

$$
g_{z}(s, k, z)-g(s, k, z)<0 \quad \forall z>0, \forall k, s \in[-1,1] .
$$

The proof of this inequality will be the goal of this section. To this aim, we compute

$$
g_{z}(s, k, z)-g(s, k, z)=W(s, k, z) \cosh (s z)+Q(s, k, z) \sinh (s z)
$$

in which we set 


$$
\begin{aligned}
W(s, k, z):= & {\left[\frac{\zeta(k, z)}{F(z)}+z \frac{\psi(k, z)}{F(z)}\right]_{z}-\left[\frac{\zeta(k, z)}{F(z)}+z \frac{\psi(k, z)}{F(z)}\right] } \\
& +s\left(\frac{\vartheta(k, z)}{\bar{F}(z)}+2 z \frac{\omega(k, z)}{\bar{F}(z)}-\left[z \frac{\omega(k, z)}{\bar{F}(z)}\right]_{z}\right)-s^{2} z \frac{\psi(k, z)}{F(z)}
\end{aligned}
$$

and

$$
\begin{aligned}
Q(s, k, z):= & {\left[\frac{\vartheta(k, z)}{\bar{F}(z)}+z \frac{\omega(k, z)}{\bar{F}(z)}\right]_{z}-\left[\frac{\vartheta(k, z)}{\bar{F}(z)}+z \frac{\omega(k, z)}{\bar{F}(z)}\right] } \\
& +s\left(\frac{\zeta(k, z)}{F(z)}+2 z \frac{\psi(k, z)}{F(z)}-\left[z \frac{\psi(k, z)}{F(z)}\right]_{z}\right)-s^{2} z \frac{\omega(k, z)}{\bar{F}(z)} .
\end{aligned}
$$

In view of the elementary implication:

$$
W(z)+|Q(z)|<0 \quad \Rightarrow \quad W(z) \cosh (v z)+Q(z) \sinh (v z)<0 \quad \forall z>0, \forall v \in \mathbb{R}
$$

for all $W, Q:(0,+\infty) \rightarrow \mathbb{R}$ continuous functions, it follows that a sufficient condition for (21) to hold is

$$
W(s, k, z)+Q(s, k, z)<0 \wedge W(s, k, z)-Q(s, k, z)<0 \quad \forall z>0, \forall k, s \in[-1,1] .
$$

We consider

$$
\begin{aligned}
W(s, k, z)+Q(s, k, z)= & -s^{2}\left[\frac{\psi(k, z)}{F(z)}+\frac{\omega(k, z)}{\bar{F}(z)}\right] \\
& +s\left(\frac{\vartheta(k, z)}{\bar{F}(z)}+\frac{\zeta(k, z)}{F(z)}+2 z \frac{\omega(k, z)}{\bar{F}(z)}+2 z \frac{\psi(k, z)}{F(z)}-\left[z \frac{\omega(k, z)}{\bar{F}(z)}+z \frac{\psi(k, z)}{F(z)}\right]_{z}\right) \\
& +\left[\frac{\zeta(k, z)}{F(z)}+z \frac{\psi(k, z)}{F(z)}+\frac{\vartheta(k, z)}{\bar{F}(z)}+z \frac{\omega(k, z)}{\bar{F}(z)}\right]_{z} \\
& -\left[\frac{\zeta(k, z)}{F(z)}+z \frac{\psi(k, z)}{F(z)}+\frac{\vartheta(k, z)}{\bar{F}(z)}+z \frac{\omega(k, z)}{\bar{F}(z)}\right] \\
W(s, k, z)-Q(s, k, z)= & -s^{2} z\left[\frac{\psi(k, z)}{F(z)}-\frac{\omega(k, z)}{\bar{F}(z)}\right] \\
& +s\left(\frac{\vartheta(k, z)}{\bar{F}(z)}-\frac{\zeta(k, z)}{F(z)}+2 z \frac{\omega(k, z)}{\bar{F}(z)}-2 z \frac{\psi(k, z)}{F(z)}-\left[z \frac{\omega(k, z)}{\bar{F}(z)}-z \frac{\psi(k, z)}{F(z)}\right]_{z}\right) \\
& +\left[\frac{\zeta(k, z)}{F(z)}+z \frac{\psi(k, z)}{F(z)}-\frac{\vartheta(k, z)}{\bar{F}(z)}-z \frac{\omega(k, z)}{\bar{F}(z)}\right]_{z} \\
& -\left[\frac{\zeta(k, z)}{F(z)}+z \frac{\psi(k, z)}{F(z)}-\frac{\vartheta(k, z)}{\bar{F}(z)}-z \frac{\omega(k, z)}{\bar{F}(z)}\right] .
\end{aligned}
$$

The maps $[-1,1] \ni s \mapsto W(s, k, z) \pm Q(s, k, z)$ are concave parabolas for all $z>0$ and $k \in[-1,1]$ fixed. Indeed, we have 


$$
\frac{\psi(k, z)}{F(z)} \pm \frac{\omega(k, z)}{\bar{F}(z)}>0 \quad \forall z>0, k \in[-1,1] .
$$

Furthermore, there hold:

$$
\left[z \frac{\psi(k, z)}{F(z)} \pm z \frac{\omega(k, z)}{\bar{F}(z)}\right]_{z}-\left[\frac{\zeta(k, z)}{F(z)} \pm \frac{\vartheta(k, z)}{\bar{F}(z)}\right]<0 \quad \forall k \in[-1,1], z>0
$$

and

$$
\left[\frac{\zeta(k, z)}{F(z)} \pm \frac{\vartheta(k, z)}{\bar{F}(z)}\right]_{z}<0 \quad \forall k \in[-1,1], z>0 .
$$

The first condition assures that the abscissa $\bar{s}$ of the parabolas vertex satisfies, respectively, $\bar{s}>1$ or $\bar{s}<-1$, implying that the maximum is achieved, respectively, at $s=1$ or at $s=-1$; condition (27) implies the negativity of such maxima proving (23) and, in turn, (21). We postpone the (long) proofs of (25), (26) and (27), respectively, to Sects. 4.3, 4.4 and 4.5.

Remark 4.1 It is worth pointing out that the proofs of (26) and (27) are achieved by repeating several times the scheme outlined above, i.e., we first put in evidence an expression of the type: $W \cosh (v z)+Q \sinh (v z)$, for suitable functions $W$ and $Q$, and then, in order to show that this expression is always negative, we exploit (22) and we come to study the sign of $W \pm Q$. As in (24), the functions $W \pm Q$ can always be seen as parabolas with respect to one of the variables: we locate the maximum point of these parabolas, and we estimate the sign of the maximum in a suitable interval. The advantage of this procedure is that, at each step, we obtain a reduction in the number of variables. Indeed, we start with the threevariable functions $W$ and $Q$ in (24) and we reduce to two or one variables functions, see e.g. (33).

Remark 4.2 Except for (26), all steps in the proof of the monotonicity issue (6) hold for all $\sigma \in(-1,1)$. Our numerical experiments suggest that (23) is still satisfied when $\sigma \in(-1,0)$ but the vertex of the parabolas $s \mapsto W(s, k, z) \pm Q(s, k, z)$ in (24), differently to what happens for $\sigma \in[0,1)$, may belong to the interval $[-1,1]$. Therefore, to extend the proof to the case $\sigma \in(-1,0)$, condition (27) should be modified accordingly.

\subsection{Proof of the positivity issue in (6).}

By (20) the sign of $\phi_{m}(y, \eta)$ is the same of the function $\phi(s, k, z)=e^{-z} g(s, k, z)+h(s, k, z)$. Since $h(s, k, z)>0$ for all $z>0, k, s \in[-1,1]$, to obtain the positivity of $\phi_{m}$ in (6) we prove that $e^{-z} g(s, k, z) \geqslant 0$ for all $z>0, k, s \in[-1,1]$. For $z \rightarrow+\infty$, by direct inspection, we get that $e^{-z} g(s, k, z) \rightarrow L$ with $L=0$ for all $k, s \neq \pm 1$ and $L=\frac{4+(1+\sigma)^{2}}{(1-\sigma)^{2}}$ if $k=s= \pm 1$. Therefore, the strict monotonicity of $e^{-z} g(s, k, z)$ proved in Sect. 4.1 assures the positivity of $\phi(z, k, s)$, i.e., the positivity issue in (6).

\subsection{Proof of inequality (25).}

Here and after, we will exploit the inequalities 


$$
\begin{gathered}
2 \cosh (v z)-(1+\sigma) \sinh (v z)>0 \quad \forall z>0, \forall v \in \mathbb{R}, \\
\frac{1}{F(z)} \pm \frac{1}{\bar{F}(z)}>0 \quad \text { and } \quad \frac{1}{[F(z)]^{2}} \pm \frac{1}{[\bar{F}(z)]^{2}}>0 \quad \forall z>0
\end{gathered}
$$

where $F(z)$ and $\bar{F}(z)$ are as in (10). The proof of (28) is immediate, while inequality (29) simply follows by noticing that $\bar{F}(z)>F(z)>0$ for all $z>0$. Next, we prove (25).

Lemma 4.3 Given $F(z), \bar{F}(z)$ as in (10) and $\psi(k, z), \omega(k, z)$ as in (18), we have

$$
\frac{\psi(k, z)}{F(z)} \pm \frac{\omega(k, z)}{\bar{F}(z)}>0 \quad \forall k \in[-1,1], z>0 .
$$

Proof Thanks to $(28)$ and $\cosh (k z)-k \sinh (k z)>0$ for all $z>0$ and $k \in[-1,1]$, we have that

$$
\begin{aligned}
\psi(k, z) & =(2+(1-\sigma) z) \cosh (k z) \cosh (z)+(-(1+\sigma)+z(1-\sigma)) \cosh (k z) \sinh (z) \\
& -k z(1-\sigma) \sinh (k z) \cosh (z)-k z(1-\sigma) \sinh (k z) \sinh (z)>0 \quad \forall k \in[-1,1], z>0 .
\end{aligned}
$$

Hence, through the first of (29), a sufficient condition for the validity of (30) is $\psi(k, z) \pm \omega(k, z)>0$. But by (28), we immediately deduce

$$
\begin{aligned}
& \psi+\omega=(2+(1-\sigma)(1-k) z) \cosh [(1+k) z]+(-(1+\sigma)+z(1-\sigma)(1-k)) \sinh [(1+k) z]>0, \\
& \psi-\omega=(2+(1-\sigma)(1+k) z) \cosh [(1-k) z]+(-(1+\sigma)+z(1-\sigma)(1+k)) \sinh [(1-k) z]>0,
\end{aligned}
$$

for all $k \in[-1,1]$ and $z>0$. This concludes the proof.

\subsection{Proof of inequality (26).}

The proof of (26) is given in Lemma 4.4.

Lemma 4.4 Given $F(z), \bar{F}(z)$ as in (10) and $\zeta(k, z), \vartheta(k, z), \psi(k, z), \omega(k, z)$ as in (18), we have that

$$
\left[z \frac{\psi}{F} \pm z \frac{\omega}{\bar{F}}\right]_{z}-\left[\frac{\zeta}{F} \pm \frac{\vartheta}{\bar{F}}\right]=\frac{\left(\psi+z \psi_{z}-\zeta\right) F-z \psi F^{\prime}}{F^{2}} \pm \frac{\left(\omega+z \omega_{z}-\vartheta\right) \bar{F}-z \omega \bar{F}^{\prime}}{\bar{F}^{2}}<0
$$

for all $k \in[-1,1]$ and $z>0$.

Proof Thanks to the symmetry properties of the functions involved, see (19), the second term of (31) is given by the sum of an even and an odd function with respect to $k$. Hence, to obtain (31), it is enough to prove that

$$
\frac{\left(\psi+z \psi_{z}-\zeta\right) F(z)-z \psi F^{\prime}}{F^{2}}+\frac{\left(\omega+z \omega_{z}-\vartheta\right) \bar{F}-z \omega \bar{F}^{\prime}}{\bar{F}^{2}}<0 \quad \forall k \in[-1,1], z>0 .
$$


We rewrite (32) as

$$
\cosh (k z) \mathcal{W}(k, z)+\sinh (k z) \mathcal{Q}(k, z)<0 \quad \forall k \in[-1,1], z>0
$$

where

$$
\mathcal{W}(k, z):=k^{2} z^{2} s(z)+k z t(z)+u(z), \quad \mathcal{Q}(k, z):=k^{2} z^{2} p(z)+k z q(z)+r(z)
$$

and

$$
\begin{aligned}
& p(z):=-\frac{(1-\sigma)}{\bar{F}(z)}[\cosh (z)+\sinh (z)]<0 \\
& q(z):= \frac{1}{F(z)}\left(2(1+\sigma) \cosh (z)-4 \sinh (z)+z(1-\sigma)[\cosh (z)+\sinh (z)] \frac{F^{\prime}(z)}{F(z)}\right) \\
& r(z):= \frac{1}{\bar{F}(z)}\left\{[\cosh (z)+\sinh (z)]\left(-\frac{2(1+\sigma)}{1-\sigma}+2 z(1-\sigma)+z^{2}(1-\sigma)\right)\right. \\
& s(z):=-\frac{(1-\sigma)}{F(z)}[\cosh (z)+\sinh (z)]<0 \\
& t(z):= \frac{1}{\bar{F}(z)}\left(-4 \cosh (z)+2(1+\sigma) \sinh (z)+z(1-\sigma)[\cosh (z)+\sinh (z)] \frac{\bar{F}^{\prime}(z)}{\bar{F}(z)}\right) \\
& u(z):= \frac{1}{F(z)}\left\{[\cosh (z)+\sinh (z)]\left(-\frac{2(1+\sigma)}{1-\sigma}+2 z(1-\sigma)+z^{2}(1-\sigma)\right)\right. \\
&\left.\quad-z(\cosh (z)[2+z(1-\sigma)]+\sinh (z)[-1-\sigma+z(1-\sigma)]) \frac{F^{\prime}(z)}{F(z)}\right\} .
\end{aligned}
$$

Clearly, the above definitions can be simplified by exploiting the identity $\cosh (z)+\sinh (z)=e^{z}$ but for the sake of computations it is convenient to keep the hyperbolic functions here and after. By (22), (33) follows if $\chi^{ \pm}(k, z):=\mathcal{W}(k, z) \pm \mathcal{Q}(k, z)<0$ for all $k \in[-1,1]$ and $z>0$, namely if

$$
\chi^{+}(k, z):=k^{2} z^{2}[s(z)+p(z)]+k z[t(z)+q(z)]+u(z)+r(z)<0 \quad \forall k \in[-1,1], z>0
$$

and

$$
\chi^{-}(k, z):=k^{2} z^{2}[s(z)-p(z)]+k z[t(z)-q(z)]+u(z)-r(z)<0 \quad \forall k \in[-1,1], z>0 .
$$

We prove the validity of (35) and (36) here below; this concludes the proof of Lemma 4.4.

Proof of (35).

By (34), $s(z)+p(z)<0$ for all $z>0$, hence $\chi^{+}(k, z)$ is a concave parabola with respect to $k$. Therefore, $\chi^{+}(k, z)<0$ if the ordinate of its vertex is negative, namely if

$$
\frac{4[s(z)+p(z)][u(z)+r(z)]-[t(z)+q(z)]^{2}}{4[s(z)+p(z)]}=: \frac{\mu(z)}{4[s(z)+p(z)]}<0 \quad \forall z>0 .
$$

Through many computations, we obtain 


$$
\begin{aligned}
\mu(z)= & 2(1-\sigma)(3+\sigma)\left[\frac{1}{[F(z)]^{2}}-\frac{1}{[\bar{F}(z)]^{2}}+2 \frac{z}{F(z) \bar{F}(z)}\left(\frac{F^{\prime}(z)}{F(z)}-\frac{\bar{F}^{\prime}(z)}{\bar{F}(z)}\right)\right] \\
& +\frac{(3+\sigma)^{2}}{[F(z) \bar{F}(z)]^{2}}\left[\cosh (2 z)\left(\left(7+10 \sigma-\sigma^{2}\right) \sinh ^{2}(2 z)-4(1-\sigma)^{2} z^{2}\right)\right. \\
& \left.+\sinh (2 z)\left(\left(7+10 \sigma-\sigma^{2}\right) \sinh ^{2}(2 z)+4(1-\sigma)^{2} z^{2}\right)\right] \\
& -[\cosh (2 z)+\sinh (2 z)] z(1-\sigma)^{2}\left(\frac{2 F(z)-F^{\prime}(z)}{[F(z)]^{2}}+\frac{2 \bar{F}(z)-\bar{F}^{\prime}(z)}{[\bar{F}(z)]^{2}}\right) \times \\
& \times\left[\frac{(4+2 z) F(z)-z F^{\prime}(z)}{[F(z)]^{2}}+\frac{(4+2 z) \bar{F}(z)-z \bar{F}^{\prime}(z)}{[\bar{F}(z)]^{2}}\right] .
\end{aligned}
$$

We have

$$
\frac{F^{\prime}(z)}{F(z)}-\frac{\bar{F}^{\prime}(z)}{\bar{F}(z)}=\frac{(3+\sigma)(1-\sigma)}{F(z) \bar{F}(z)}[2 z \cosh (2 z)-\sinh (2 z)]>0 \quad \forall z>0
$$

since $[2 z \cosh (2 z)-\sinh (2 z)]^{\prime}(z)=4 z \sinh (2 z)>0$ for all $z>0$. Hence, recalling (29), the first term in the definition of $\mu$ is positive. Moreover, by estimating $\sinh (2 z)>2 z$ for $z>0$, we have

$$
\begin{gathered}
\left(7+10 \sigma-\sigma^{2}\right) \sinh ^{2}(2 z)-4(1-\sigma)^{2} z^{2}>8 z^{2}(\sigma+2 \sqrt{3}-3)(3+2 \sqrt{3}-\sigma)>0 \quad \forall z>0 \\
\left(7+10 \sigma-\sigma^{2}\right) \sinh ^{2}(2 z)+4(1-\sigma)^{2} z^{2}=(\sigma+4 \sqrt{2}-5)(5+4 \sqrt{2}-\sigma) \sinh ^{2}(2 z) \\
+4(1-\sigma)^{2} z^{2}>0 \quad \forall z>0 .
\end{gathered}
$$

By Lemma 6.1 in "Appendix," we know that $\frac{2 F(z)-F^{\prime}(z)}{[F(z)]^{2}}+\frac{2 \bar{F}(z)-\bar{F}^{\prime}(z)}{[\bar{F}(z)]^{2}}<0$, therefore if

$$
\frac{(4+2 z) F(z)-z F^{\prime}(z)}{[F(z)]^{2}}+\frac{(4+2 z) \bar{F}(z)-z \bar{F}^{\prime}(z)}{[\bar{F}(z)]^{2}}>0 \quad \forall z>0,
$$

then $\mu(z)>0$. To this aim, we consider

$$
\mu_{1}(z):=(4+2 z) F(z)-z F^{\prime}(z)=(3+\sigma)\{z[\sinh (2 z)-\cosh (2 z)]+2 \sinh (2 z)\}-(1-\sigma)\left(3 z+2 z^{2}\right) ;
$$

since $\mu_{1}(0)=0$ and

$$
\begin{aligned}
\mu_{1}^{\prime}(z) & =(3+\sigma)\{\cosh (2 z)[3+2 z]+\sinh (2 z)[1-2 z]\}-(1-\sigma)(3+4 z) \\
& >2\{(3+\sigma) z[\cosh (2 z)-\sinh (2 z)]+3+3 \sigma+z(1+3 \sigma)\}>0 \quad \forall z>0,
\end{aligned}
$$

we have $\mu_{1}(z)>0$ for all $z>0$. On the other hand, for all $z>0$, we have

$$
\begin{gathered}
\mu_{2}(z):=(4+2 z) \bar{F}(z)-z \bar{F}^{\prime}(z)=(3+\sigma)\{z[\sinh (2 z)-\cosh (2 z)]+2 \sinh (2 z)\} \\
+(1-\sigma)\left(3 z+2 z^{2}\right)>\mu_{1}(z)>0,
\end{gathered}
$$

implying (37). This assures $\chi^{+}(k, z)<0$ for all $k \in[-1,1]$ and for all $z>0$.

Proof of (36).

First of all, we notice that $\chi^{-}(k, z)$ is a concave parabola with respect to $k$, since 


$$
s(z)-p(z)=-(1-\sigma)\left[\frac{1}{F(z)}-\frac{1}{\bar{F}(z)}\right][\cosh (z)+\sinh (z)]<0 \quad \forall z>0 .
$$

We prove that the parabola has a point of maximum for $k<-1$, i.e., that

$$
\bar{\mu}(z):=t(z)-q(z)+2 z[p(z)-s(z)]<0 \quad \forall z>0 .
$$

To this aim, we study

$$
\begin{aligned}
\bar{\mu}(z)= & \frac{2}{F(z) \bar{F}(z)}[\sinh (z)[(1+\sigma) F(z)+2 \bar{F}(z)]-\cosh (z)[2 F(z)+(1+\sigma) \bar{F}(z)]] \\
& +z(1-\sigma)[\cosh (z)+\sinh (z)]\left[\frac{2 F(z)-F^{\prime}(z)}{[F(z)]^{2}}-\frac{2 \bar{F}(z)-\bar{F}^{\prime}(z)}{[\bar{F}(z)]^{2}}\right] .
\end{aligned}
$$

By Lemma 6.1 in "Appendix," we have that the last term above is negative; about the remaining terms, we distinguish the cases $z \in(0,1]$ and $z>1$. For $z \in(0,1]$, we have

$$
\begin{aligned}
& \sinh (z)[(1+\sigma) F(z)+2 \bar{F}(z)]-\cosh (z)[2 F(z)+(1+\sigma) \bar{F}(z)] \\
& \quad=\frac{(3+\sigma)^{2}}{2} \sinh (2 z)[\sinh (z)-\cosh (z)]+z(1-\sigma)^{2}[\cosh (z)+\sinh (z)] \\
& \quad<2 z\left[\left(\sigma^{2}+2 \sigma+5\right) \sinh (z)-4(1+\sigma) \cosh (z)\right]:=v(\sigma)
\end{aligned}
$$

We observe that $\frac{d v}{d \sigma}=4 z[(1+\sigma) \sinh (z)-2 \cosh (z)]<0$; $\quad$ hence, $v(\sigma)<2 z[5 \sinh (z)-4 \cosh (z)]<0$ for $z<\log 3$, implying $\bar{\mu}(z)<0$ for $z \in(0,1]$. For $z>1$, we rewrite

$$
\bar{\mu}(z)=\cosh (z) \bar{W}(z)+\sinh (z) \bar{Q}(z),
$$

where

$$
\begin{aligned}
& \bar{W}(z):=-\frac{4}{\bar{F}(z)}-\frac{2(1+\sigma)}{F(z)}+z(1-\sigma)\left(\frac{2 F(z)-F^{\prime}(z)}{[F(z)]^{2}}-\frac{2 \bar{F}(z)-\bar{F}^{\prime}(z)}{[\bar{F}(z)]^{2}}\right) \\
& \bar{Q}(z):=\frac{2(1+\sigma)}{\bar{F}(z)}+\frac{4}{F(z)}+z(1-\sigma)\left(\frac{2 F(z)-F^{\prime}(z)}{[F(z)]^{2}}-\frac{2 \bar{F}(z)-\bar{F}^{\prime}(z)}{[\bar{F}(z)]^{2}}\right)
\end{aligned}
$$

and, by (22), we prove that $\bar{\mu}$ is negative by showing that $\bar{W}(z) \pm \bar{Q}(z)<0$ for $z>1$.

The case $\bar{W}(z)-\bar{Q}(z)<0$ is trivially true for all $z>0$; then, it remains to study

$$
\bar{W}(z)+\bar{Q}(z)=z \frac{(1-\sigma)^{2}}{[F(z) \bar{F}(z)]^{2}}\left[(3+\sigma)^{2}((1+z) \cosh (4 z)-2 z \sinh (4 z)-1-z)-8 z^{3}(1-\sigma)^{2}\right] .
$$

We consider $\bar{\mu}_{1}(z):=(1+z) \cosh (4 z)-2 z \sinh (4 z)-1$ and $\bar{\mu}_{1}^{\prime}(z)=(1-8 z) \cosh (4 z)$ $+2(1+2 z) \sinh (4 z)$, so that $\bar{\mu}_{1}(z)$ has stationary points satisfying $\tanh (4 \bar{z})=(8 \bar{z}-1) /$ $[2(1+2 \bar{z})]:=\gamma(\bar{z})$. We observe that $\gamma(\bar{z})$ is always increasing for $\bar{z}>0, \gamma(\bar{z})=1$ if and only if $\bar{z}=3 / 4<1$, implying $\bar{\mu}_{1}^{\prime}(z)<0$ for $z>1$; since $\bar{\mu}_{1}(1)=2 e^{-4}-1<0$, then $\bar{\mu}_{1}(z)<0$ for $z>1$ and in conclusion $\bar{W}(z)+\bar{Q}(z)<0$ for all $z>1$. This proves (38). In view of (38), to obtain $\chi^{-}(k, z)<0$ for all $k \in[-1,1]$ and $z>0$, it is enough to study the sign of 


$$
\begin{aligned}
\chi^{-}(-1, z)= & z^{2}[s(z)-p(z)]-z[t(z)-q(z)]+u(z)-r(z) \\
= & -\frac{2(1+\sigma)}{1-\sigma}\left(\frac{1}{F(z)}-\frac{1}{\bar{F}(z)}\right)[\cosh (z)+\sinh (z)] \\
& +z\left\{\cosh (z)\left[2 \frac{2 F(z)-F^{\prime}(z)}{[F(z)]^{2}}+(1+\sigma) \frac{2 \bar{F}(z)-\bar{F}^{\prime}(z)}{[\bar{F}(z)]^{2}}\right]\right. \\
& \left.\quad-\sinh (z)\left[(1+\sigma) \frac{2 F(z)-F^{\prime}(z)}{[F(z)]^{2}}+2 \frac{2 \bar{F}(z)-\bar{F}^{\prime}(z)}{[\bar{F}(z)]^{2}}\right]\right\} \\
= & -\frac{2(1+\sigma)}{1-\sigma}\left(\frac{1}{F(z)}-\frac{1}{\bar{F}(z)}\right)[\cosh (z)+\sinh (z)]+z\{\cosh (z) \widetilde{W}(z)+\sinh (z) \widetilde{Q}(z)\}
\end{aligned}
$$

where

$$
\widetilde{W}(z):=2 \frac{2 F(z)-F^{\prime}(z)}{[F(z)]^{2}}+(1+\sigma) \frac{2 \bar{F}(z)-\bar{F}^{\prime}(z)}{[\bar{F}(z)]^{2}}
$$

and

$$
\widetilde{Q}(z):=-(1+\sigma) \frac{2 F(z)-F^{\prime}(z)}{[F(z)]^{2}}-2 \frac{2 \bar{F}(z)-\bar{F}^{\prime}(z)}{[\bar{F}(z)]^{2}} .
$$

Recalling that $\bar{F}(z)>F(z)>0$ for all $z>0$, if

$$
\cosh (z) \widetilde{W}(z)+\sinh (z) \widetilde{Q}(z)<0 \quad \forall z>0,
$$

we conclude that $\bar{\chi}(-1, z)<0$ and the thesis. By (22), this follows by showing that $\widetilde{W}(z) \pm \widetilde{Q}(z)<0$ for all $z>0$, namely that

$$
(1-\sigma)\left[\frac{2 F(z)-F^{\prime}(z)}{[F(z)]^{2}}-\frac{2 \bar{F}(z)-\bar{F}^{\prime}(z)}{[\bar{F}(z)]^{2}}\right]<0 \quad(3+\sigma)\left[\frac{2 F(z)-F^{\prime}(z)}{[F(z)]^{2}}+\frac{2 \bar{F}(z)-\bar{F}^{\prime}(z)}{[\bar{F}(z)]^{2}}\right]<0 .
$$

These inequalities hold true for all $z>0$ thanks to Lemma 6.1 in "Appendix".

\subsection{Proof of inequality (27).}

The proof of (27) is given in Lemma 4.5.

Lemma 4.5 Given $F(z), \bar{F}(z)$ as in (10) and $\zeta(k, z), \vartheta(k, z)$ as in(18), we have

$$
\left[\frac{\zeta}{F} \pm \frac{\vartheta}{\bar{F}}\right]_{z}=\frac{\zeta_{z} F-\zeta F^{\prime}}{[F]^{2}} \pm \frac{\vartheta_{z} \bar{F}-\vartheta \bar{F}^{\prime}}{[\bar{F}]^{2}}<0 \quad \forall k \in[-1,1], z>0
$$


Proof Thanks to the symmetry properties of the functions involved, see (19), the second term of (39) is given by the sum of an even and an odd function with respect to $k$; hence, to obtain (39), it is enough to prove that

$$
\frac{\zeta_{z}(k, z) F(z)-\zeta(k, z) F^{\prime}(z)}{[F(z)]^{2}}+\frac{\vartheta_{z}(k, z) \bar{F}(z)-\vartheta(k, z) \bar{F}^{\prime}(z)}{[\bar{F}(z)]^{2}}<0,
$$

for all $k \in[-1,1]$ and $z>0$. We rewrite (40) as

$$
\cosh (k z) \mathcal{V}(k, z)+\sinh (k z) \mathcal{P}(k, z)
$$

where

$$
\mathcal{V}(k, z):=k^{2} a(z)+k b(z)+c(z), \quad \mathcal{P}(k, z):=k^{2} d(z)+k e(z)+f(z),
$$

and

$$
\begin{aligned}
& a(z):=-\frac{z}{F(z)}[2 \cosh (z)-(1+\sigma) \sinh (z)]<0 \\
& b(z):= \frac{1}{\bar{F}(z)}\left(\frac{2(1+\sigma)}{1-\sigma}[\cosh (z)+\sinh (z)]+z[2 \sinh (z)-(1+\sigma) \cosh (z)] \frac{\bar{F}^{\prime}(z)}{\bar{F}(z)}\right) \\
& c(z):= \frac{1}{F(z)}\left\{\cosh (z)\left(\frac{2 \sigma(1+\sigma)}{1-\sigma}+2 z\right)+\sinh (z)\left(\frac{2(3-\sigma)}{1-\sigma}-z(1+\sigma)\right)\right. \\
&\left.-\left[\cosh (z)\left(\frac{4}{1-\sigma}-z(1+\sigma)\right)+\sinh (z)\left(\frac{(1+\sigma)^{2}}{1-\sigma}+2 z\right)\right] \frac{F^{\prime}(z)}{F(z)}\right\} \\
& d(z):=-\frac{z}{\bar{F}(z)}[2 \sinh (z)-(1+\sigma) \cosh (z)] \\
& e(z):=\frac{1}{F(z)}\left(\frac{2(1+\sigma)}{1-\sigma}[\cosh (z)+\sinh (z)]+z[2 \cosh (z)-(1+\sigma) \sinh (z)] \frac{F^{\prime}(z)}{F(z)}\right) \\
& f(z):=\frac{1}{\bar{F}(z)}\left\{\cosh (z)\left(\frac{2(3-\sigma)}{1-\sigma}-z(1+\sigma)\right)+\sinh (z)\left(\frac{2 \sigma(1+\sigma)}{1-\sigma}+2 z\right)\right. \\
&\left.\quad-\left[\cosh (z)\left(\frac{(1+\sigma)^{2}}{1-\sigma}+2 z\right)+\sinh (z)\left(\frac{4}{1-\sigma}-z(1+\sigma)\right)\right] \frac{\bar{F}^{\prime}(z)}{\bar{F}(z)}\right\} .
\end{aligned}
$$

Then, by (22), we obtain the thesis if $\Xi(k, z)^{ \pm}:=\mathcal{V}(k, z) \pm \mathcal{P}(k, z)<0$ for all $k \in[-1,1]$ and $z>0$, i.e., if

$$
\Xi^{+}(k, z):=k^{2}[a(z)+d(z)]+k[b(z)+e(z)]+c(z)+f(z)<0 \quad \forall k \in[-1,1], z>0
$$

and

$$
\Xi^{-}(k, z):=k^{2}[a(z)-d(z)]+k[b(z)-e(z)]+c(z)-f(z)<0 \quad \forall k \in[-1,1], z>0 .
$$

We prove the validity of (42) and (43) here below. This concludes the proof of Lemma 4.5.

Proof of (42).

We consider 


$$
a(z)+d(z)=-z\left[\cosh (z)\left(\frac{2}{F(z)}-\frac{(1+\sigma)}{\bar{F}(z)}\right)+\sinh (z)\left(\frac{2}{\bar{F}(z)}-\frac{(1+\sigma)}{F(z)}\right)\right] .
$$

Since, from (29), we have

$$
\frac{2}{F(z)}-\frac{(1+\sigma)}{\bar{F}(z)} \pm\left(\frac{2}{\bar{F}(z)}-\frac{(1+\sigma)}{F(z)}\right)=[2 \mp(1+\sigma)]\left(\frac{1}{F(z)} \pm \frac{1}{\bar{F}(z)}\right)>0 \quad \forall z>0,
$$

by (22) we infer that $a(z)+d(z)<0$; hence, the map $k \mapsto \Xi^{+}(k, z)$ is a concave parabola for all $z>0$. Now we prove that the parabola has the abscissa vertex at $k=\bar{k}$ with $\bar{k}>1$; this follows by showing that

$$
b(z)+e(z)+2[a(z)+d(z)]>0 \quad \forall z>0 .
$$

We have

$$
\begin{aligned}
b(z)+e(z)+2[a(z)+d(z)]= & \frac{2(1+\sigma)}{(1-\sigma)}[\cosh (z)+\sinh (z)]\left(\frac{1}{F(z)}+\frac{1}{\bar{F}(z)}\right) \\
& +z\left\{\cosh (z)\left[2\left(\frac{F^{\prime}(z)}{F(z)^{2}}-\frac{2}{F(z)}\right)-(1+\sigma)\left(\frac{\bar{F}^{\prime}(z)}{\bar{F}(z)^{2}}-\frac{2}{\bar{F}(z)}\right)\right]\right. \\
& \left.+\sinh (z)\left[2\left(\frac{\bar{F}^{\prime}(z)}{\bar{F}(z)^{2}}-\frac{2}{\bar{F}(z)}\right)-(1+\sigma)\left(\frac{F^{\prime}(z)}{F(z)^{2}}-\frac{2}{F(z)}\right)\right]\right\} .
\end{aligned}
$$

Through (22), (44) holds if

$$
[2 \mp(1+\sigma)]\left(\frac{F^{\prime}(z)-2 F(z)}{F(z)^{2}} \pm \frac{\bar{F}^{\prime}(z)-2 \bar{F}(z)}{\bar{F}(z)^{2}}\right)>0 ;
$$

this condition is guaranteed for all $z>0$ by Lemma 6.1 in "Appendix". Hence, the maximum of $\Xi^{+}(k, z)$ is achieved at $k=1$; we prove that $\Xi^{+}(1, z)=a(z)+d(z)+b(z)+e(z)+c(z)+f(z)<0$ for all $z>0$. To this aim, we consider

$$
\begin{aligned}
\Xi^{+}(1, z)= & \frac{1}{F(z)^{2}}\left\{\cosh (z)\left[\frac{2(1+\sigma)^{2}}{1-\sigma} F(z)+F^{\prime}(z)\left(z(3+\sigma)-\frac{4}{1-\sigma}\right)\right]\right. \\
& \left.+\sinh (z)\left[\frac{8}{1-\sigma} F(z)-F^{\prime}(z)\left(z(3+\sigma)+\frac{(1+\sigma)^{2}}{1-\sigma}\right)\right]\right\} \\
& +\frac{1}{\bar{F}(z)^{2}}\left\{\cosh (z)\left[\frac{8}{1-\sigma} \bar{F}(z)-\bar{F}^{\prime}(z)\left(z(3+\sigma)+\frac{(1+\sigma)^{2}}{1-\sigma}\right)\right]\right. \\
& \left.+\sinh (z)\left[\frac{2(1+\sigma)^{2}}{1-\sigma} \bar{F}(z)+\bar{F}^{\prime}(z)\left(z(3+\sigma)-\frac{4}{1-\sigma}\right)\right]\right\} \\
:= & \frac{\varsigma(z)}{F(z)^{2}}+\frac{\bar{\zeta}(z)}{\bar{F}(z)^{2}} .
\end{aligned}
$$

Recalling that $\bar{F}(z)>F(z)>0$ for all $z>0$, we obtain that $\Xi^{+}(1, z)<0$ by showing that $\varsigma(z)<0$ and $\varsigma(z)+\bar{\varsigma}(z)<0$. Through many computations, we get 


$$
\varsigma(z)=\frac{e^{z}}{2}\left[-(1-\sigma)^{2} z-4(1+\sigma)-4 \frac{(3+\sigma)(1+\sigma)}{1-\sigma} e^{-2 z}+(3+\sigma)^{2} z e^{-4 z}\right] .
$$

Considering $\quad \tilde{\varsigma}(z):=-(1-\sigma)^{2} z-4(1+\sigma)+(3+\sigma)^{2} z e^{-4 z}, \quad$ we have that $\widetilde{\varsigma}(0)=-4(1+\sigma)<0 \quad$ and $\quad \tilde{\zeta}(z) \rightarrow-\infty \quad$ as $\quad z \rightarrow+\infty$; furthermore, $\widetilde{\varsigma}^{\prime}(z)=-(1-\sigma)^{2}+(3+\sigma)^{2} e^{-4 z}(1-4 z) \quad$ so that $\tilde{\varsigma}^{\prime}(\bar{z})=0$ if and only if $\bar{z} e^{-4 \bar{z}}=\frac{e^{-4 \bar{z}}}{4}-\frac{(1-\sigma)^{2}}{4(3+\sigma)^{2}}$. We have

$$
\widetilde{\varsigma}(\bar{z})=-(1-\sigma)^{2}\left(\bar{z}+\frac{1}{4}\right)-4(1+\sigma)+(3+\sigma)^{2} \frac{e^{-4 \bar{z}}}{4} .
$$

Since $-(1-\sigma)^{2}\left(\bar{z}+\frac{1}{4}\right)-4(1+\sigma)+(3+\sigma)^{2} \frac{e^{-4 \bar{z}}}{4}<0$ for all $z>0$, we infer that $\widetilde{\varsigma}(\bar{z})<0$ and, in turn, that

$$
\widetilde{\varsigma}(z)=-(1-\sigma)^{2} z-4(1+\sigma)+(3+\sigma)^{2} z e^{-4 z}<0 \quad \forall z>0 .
$$

This yields $\varsigma(z)<0$ for all $z>0$. On the other hand, we have $\varsigma(z)+\bar{\varsigma}(z)=-4 \frac{(1+\sigma)(3+\sigma)}{1-\sigma} e^{-z}<0$ for all $z>0$. By this, we conclude that $\Xi^{+}(1, z)<0$ for all $z>0$ and, in turn, that $\Xi^{+}(k, z)<0$ for all $k \in[-1,1]$ and $z>0$.

Proof of (42).

We have

$$
a(z)-d(z)=-z\left[\cosh (z)\left(\frac{2}{F(z)}+\frac{(1+\sigma)}{\bar{F}(z)}\right)-\sinh (z)\left(\frac{2}{\bar{F}(z)}+\frac{(1+\sigma)}{F(z)}\right)\right] .
$$

Since

$$
\frac{2}{F(z)}+\frac{(1+\sigma)}{\bar{F}(z)} \pm\left(\frac{2}{\bar{F}(z)}+\frac{(1+\sigma)}{F(z)}\right)=[2 \pm(1+\sigma)]\left(\frac{1}{F(z)} \pm \frac{1}{\bar{F}(z)}\right)>0 \quad \forall z>0,
$$

by (22), we infer that $a(z)-d(z)<0$ and the map $k \mapsto \Xi^{-}(k, z)$ is a concave parabola for all $z>0$. Now we prove that the abscissa $\bar{k}$ of the parabola vertex satisfies $\bar{k}<-1$, namely that

$$
b(z)-e(z)+2[d(z)-a(z)]<0 \quad \forall z>0 .
$$

We have that

$$
\begin{aligned}
b(z)-e(z)+2[d(z)-a(z)]= & -\frac{2(1+\sigma)}{(1-\sigma)}[\cosh (z)+\sinh (z)]\left(\frac{1}{F(z)}-\frac{1}{\bar{F}(z)}\right) \\
& +z\left\{\operatorname { c o s h } ( z ) \left[2\left(\frac{2}{F(z)}-\frac{F^{\prime}(z)}{F(z)^{2}}\right)+(1+\sigma)\left(\frac{2}{\bar{F}(z)}-\frac{\bar{F}^{\prime}(z)}{\left.\left.\bar{F}(z)^{2}\right)\right]}\right.\right.\right. \\
& \left.+\sinh (z)\left[-2\left(\frac{2}{\bar{F}(z)}-\frac{\bar{F}^{\prime}(z)}{\bar{F}(z)^{2}}\right)-(1+\sigma)\left(\frac{2}{F(z)}-\frac{F^{\prime}(z)}{F(z)^{2}}\right)\right]\right\} .
\end{aligned}
$$

Through (22), (46) follows if

$$
[2 \pm(1+\sigma)]\left(\frac{2 F(z)-F^{\prime}(z)}{F(z)^{2}} \pm \frac{2 \bar{F}(z)-\bar{F}^{\prime}(z)}{\bar{F}(z)^{2}}\right)<0
$$


this condition is guaranteed for all $z>0$ by Lemma 6.1 in "Appendix". Hence, by (46), $\Xi^{-}(k, z)$ achieves its maximum at $k=-1$; we prove that $\Xi^{-}(-1, z)=a(z)-d(z)-b(z)+e(z)+c(z)-f(z)<0$ for all $z>0$. To this aim, we consider

$$
\begin{aligned}
\Xi^{-}(-1, z)= & \frac{1}{F(z)^{2}}\left\{\cosh (z)\left[\frac{2(1+\sigma)^{2}}{1-\sigma} F(z)+F^{\prime}(z)\left(z(3+\sigma)-\frac{4}{1-\sigma}\right)\right]\right. \\
& \left.+\sinh (z)\left[\frac{8}{1-\sigma} F(z)-F^{\prime}(z)\left(z(3+\sigma)+\frac{(1+\sigma)^{2}}{1-\sigma}\right)\right]\right\} \\
& -\frac{1}{\bar{F}(z)^{2}}\left\{\cosh (z)\left[\frac{8}{1-\sigma} \bar{F}(z)-\bar{F}^{\prime}(z)\left(z(3+\sigma)+\frac{(1+\sigma)^{2}}{1-\sigma}\right)\right]\right. \\
& \left.+\sinh (z)\left[\frac{2(1+\sigma)^{2}}{1-\sigma} \bar{F}(z)+\bar{F}^{\prime}(z)\left(z(3+\sigma)-\frac{4}{1-\sigma}\right)\right]\right\} \\
:= & \frac{\varsigma(z)}{F(z)^{2}}-\frac{\bar{\zeta}(z)}{\bar{F}(z)^{2}}
\end{aligned}
$$

where $\varsigma(z)$ and $\bar{\zeta}(z)$ are as defined in the proof of (42). We have already proved that $\varsigma(z)<0$ for all $z>0$, by (45) we also get $\varsigma(z)-\bar{\varsigma}(z)=e^{z} \widetilde{\varsigma}(z)<0$ for all $z>0$.

Hence, through (29) we deduce that $\Xi^{-}(-1, z)<0$ for all $z>0$. This assures $\Xi^{-}(k, z)<0$ for all $k \in[-1,1], z>0$ and concludes the proof of (43).

\section{Proof of Theorem 2.2}

The proof is achieved by showing the positivity of the Green function $G_{p}(q)$ for $p$ and $q$ belonging to suitable rectangles or union of rectangles covering $\widetilde{\Omega}$. By Theorem 2.1, we know that

$$
G_{p}(q)=\sum_{m=1}^{+\infty} \frac{1}{2 \pi} \frac{\phi_{m}(y, \eta)}{m^{3}} \sin (m \xi) \sin (m x) \quad \forall q=(x, y) \in \bar{\Omega}, \quad \forall p=(\xi, \eta) \in \bar{\Omega} .
$$

In this section, we will omit the dependence of $\phi_{m}$ from $y$ and $\eta$, implying that all relations we write hold true for all $y, \eta \in[-d, d]$; for this reason and for brevity, we will write $G(x, \xi)$ instead of $G_{p}(q)=G(x, y, \xi, \eta)$. Furthermore, we will repeatedly exploit the fact that

$$
G(x, \xi)=G(\xi, x) \quad \forall(x, \xi) \in[0, \pi] \times[0, \pi] .
$$

We start by showing the positivity of $G_{p}(q)$ for $p$ or $q$ far from the hinged edges of $\Omega$.

Proposition 5.1 There holds

$$
G(x, \xi)>0 \quad \forall(x, \xi) \in\left[\frac{\pi}{4}, \frac{3}{4} \pi\right] \times(0, \pi) \quad \text { and } \quad \forall(x, \xi) \in(0, \pi) \times\left[\frac{\pi}{4}, \frac{3}{4} \pi\right] .
$$

Proof Thanks to Theorem 2.1-(6), we know that 


$$
0<\phi_{m}<\phi_{1} \quad \forall m>1 .
$$

Noting that $|\sin (m \xi)|<m \sin (\xi)$ for all $\xi \in(0, \pi)$, see, e.g., [13], we obtain

$$
\frac{\phi_{m}}{m^{3}}|\sin (m \xi) \sin (m x)| \leqslant \frac{\phi_{1}}{m^{2}} \sin (\xi)|\sin (m x)| \quad \forall x, \xi \in(0, \pi),
$$

from which

$$
\sum_{m=2}^{\infty} \frac{\phi_{m}}{m^{3}}|\sin (m \xi) \sin (m x)| \leqslant \phi_{1} \sin (\xi) \sum_{m=2}^{\infty} \frac{|\sin (m x)|}{m^{2}} \quad \forall x, \xi \in(0, \pi) .
$$

Since

$$
\sum_{m=2}^{\infty} \frac{|\sin (m x)|}{m^{2}} \leqslant \sum_{m=2}^{\infty} \frac{1}{m^{2}}=\frac{\pi^{2}}{6}-1 \quad \forall x \in(0, \pi)
$$

we infer that

$$
\sum_{m=2}^{\infty} \frac{\phi_{m}}{m^{3}} \sin (m \xi) \sin (m x) \geqslant-\phi_{1} \sin (\xi)\left(\frac{\pi^{2}}{6}-1\right) \quad \forall x, \xi \in(0, \pi)
$$

and, in turn, that

$$
G(x, \xi) \geqslant \frac{\phi_{1}}{2 \pi} \sin (\xi)\left[\sin (x)-\left(\frac{\pi^{2}}{6}-1\right)\right] \quad \forall x, \xi \in(0, \pi) .
$$

We denote by

$$
x_{1}:=\arcsin \left(\frac{\pi^{2}}{6}-1\right) \approx 0.70<\frac{\pi}{4}
$$

hence, through (48) we have $G(x, \xi)>0$ for all $(x, \xi) \in\left(x_{1}, \pi-x_{1}\right) \times(0, \pi)$, implying

$$
G(x, \xi)>0 \quad \forall(x, \xi) \in\left[\frac{\pi}{4}, \frac{3}{4} \pi\right] \times(0, \pi) .
$$

The positivity in the region $(x, \xi) \in(0, \pi) \times\left[\frac{\pi}{4}, \frac{3}{4} \pi\right]$ follows by (47).

Our next aim is to show the positivity issue for both $p$ and $q$ near the same hinged edge, i.e., near $x=0$ and $\xi=0$ or near $x=\pi$ and $\xi=\pi$. The first step of the proof is given by the following:

Lemma 5.2 Fixing $N \geqslant 3$ integer, there holds

$$
G(x, \xi)>0 \quad \forall(x, \xi) \in\left[\frac{\pi}{N+2}, \frac{\pi}{N+1}\right) \times\left(0, \frac{\pi}{N+1}\right)
$$

and 


$$
G(x, \xi)>0 \quad \forall(x, \xi) \in\left(0, \frac{\pi}{N+1}\right) \times\left[\frac{\pi}{N+2}, \frac{\pi}{N+1}\right) .
$$

Proof We fix $N \geqslant 3$ and we rewrite $G(x, \xi)$ as follows

$$
G(x, \xi)=\frac{1}{2 \pi} \sum_{m=1}^{N} \frac{\phi_{m}}{m^{3}} \sin (m x) \sin (m \xi)+\frac{1}{2 \pi} \sum_{m=N+1}^{\infty} \frac{\phi_{m}}{m^{3}} \sin (m x) \sin (m \xi) .
$$

Then, we exploit the elementary inequality

$$
\sin (m x) \sin (m \xi)>\sin (x) \sin (\xi) \quad \forall x, \xi \in\left(0, \frac{\pi}{N+1}\right), \quad \forall m=2, \ldots, N
$$

(see Lemma 6.2 in "Appendix" for a proof) and Theorem 2.1-(6) to get

$$
\sum_{m=1}^{N} \frac{\phi_{m}}{m^{3}} \sin (m x) \sin (m \xi)>\phi_{N} \sin (x) \sin (\xi) \sum_{m=1}^{N} \frac{1}{m^{3}} \quad \forall x, \xi \in\left(0, \frac{\pi}{N+1}\right) .
$$

On the other hand, through $|\sin (m \xi)|<m \sin (\xi)$ for all $\xi \in(0, \pi)$ and Theorem 2.1-(6), we get

$$
\left|\sum_{m=N+1}^{\infty} \frac{\phi_{m}}{m^{3}} \sin (m x) \sin (m \xi)\right| \leqslant \sum_{m=N+1}^{\infty} \frac{\phi_{m}}{m^{3}}|\sin (m x) \sin (m \xi)| \leqslant \phi_{N} \sin (\xi) \sum_{m=N+1}^{\infty} \frac{1}{m^{2}} .
$$

By combining (49) and (50), we infer

$$
G(x, \xi) \geqslant \frac{\phi_{N}}{2 \pi} \sin (\xi)\left(\sum_{m=1}^{N} \frac{1}{m^{3}}\right)\left[\sin (x)-C_{N}\right] \quad \forall x, \xi \in\left(0, \frac{\pi}{N+1}\right),
$$

where

$$
C_{N}:=\frac{\sum_{m=N+1}^{\infty} \frac{1}{m^{2}}}{\sum_{m=1}^{N} \frac{1}{m^{3}}} .
$$

Next, we denote by $x_{N}$ the unique solution to the equation

$$
\sin \left(x_{N}\right)=C_{N} \quad x_{N} \in(0, \pi / 2) ;
$$

the above definition makes sense for all $N \geqslant 1$ since the map $N \mapsto C_{N}$ is positive, strictly decreasing and $0<C_{N}<1$. We prove that

$$
x_{N}<\frac{\pi}{N+2} \quad \forall N \geqslant 3 .
$$

When $N=3$, we have $x_{3} \approx 0.25<\frac{\pi}{5}$ and (53) follows. We complete the proof of (53) by showing that 


$$
C_{N}<\sin \left(\frac{\pi}{N+2}\right) \quad \forall N \geqslant 4
$$

To this purpose, we write some estimates on the numerical series; it is easy to see that

$$
\sum_{m=1}^{N} \frac{1}{m^{3}}>1 \quad \text { and } \quad \sum_{m=N+1}^{\infty} \frac{1}{m^{2}} \leqslant \int_{N}^{\infty} \frac{1}{x^{2}} d x=\frac{1}{N} \quad \forall N \geqslant 2,
$$

implying

$$
C_{N}<\frac{1}{N} \quad \forall N \geqslant 2
$$

To tackle (54), we use the estimate:

$$
\sin (x) \geqslant \frac{3}{\pi} x \quad \forall x \in\left(0, \frac{\pi}{6}\right]
$$

Combining (55) and (56), (54) follows by noticing that $\frac{1}{N}<\frac{3}{N+2}$ for all $N \geqslant 4$. Finally, in view of (53), the first part of the statement of Lemma 5.2 readily comes from the positivity of the r.h.s. of (51). Instead, the second part of the statement follows by exploiting (47).

Finally, from Lemma 5.2, we get

Proposition 5.3 There holds

$$
G(x, \xi)>0 \quad \forall(x, \xi) \in\left(0, \frac{\pi}{4}\right) \times\left(0, \frac{\pi}{4}\right) \quad \text { and } \quad \forall(x, \xi) \in\left(\frac{3}{4} \pi, \pi\right) \times\left(\frac{3}{4} \pi, \pi\right) .
$$

Proof Fixing $N \geqslant 3$ integer, from Lemma 5.2, we get

$$
G(x, \xi)>0 \quad \forall(x, \xi) \in\left(0, \frac{\pi}{N+1}\right)^{2} \backslash\left(0, \frac{\pi}{N+2}\right)^{2}
$$

and taking the infinite union of the above sets, we get

$$
G(x, \xi)>0 \quad \forall(x, \xi) \in \bigcup_{N=3}^{\infty}\left(0, \frac{\pi}{N+1}\right)^{2} \backslash\left(0, \frac{\pi}{N+2}\right)^{2}=\left(0, \frac{\pi}{4}\right)^{2}
$$

which is the first part of the statement of Proposition 5.3. To complete the proof, we set $\bar{x}=\pi-x, \bar{\xi}=\pi-\xi$ and we observe that

$$
G(x, \xi)=G(\pi-\bar{x}, \pi-\bar{\xi})=G(\bar{x}, \bar{\xi}) \quad \forall x, \xi \in\left(\frac{3}{4} \pi, \pi\right), \forall \bar{x}, \bar{\xi} \in\left(0, \frac{\pi}{4}\right) .
$$

Then, the thesis comes from (57) written for $\bar{x}$ and $\bar{\xi}$.

It remains to study the sign of the Green function for $p$ and $q$ near to opposite hinged edges, i.e., near $x=0$ and $\xi=\pi$ or near $x=\pi$ and $\xi=0$. At first, we prove the following: 
Lemma 5.4 Fixing $N \geqslant 3$, odd integer, there hold

$$
G(x, \xi)>0 \quad \forall(x, \xi) \in\left[\frac{\pi}{N+3}, \frac{\pi}{N+1}\right) \times\left(\pi-\frac{\pi}{N+1}, \pi\right)
$$

and

$$
G(x, \xi)>0 \quad \forall(x, \xi) \in\left(0, \frac{\pi}{N+1}\right) \times\left(\pi-\frac{\pi}{N+1}, \pi-\frac{\pi}{N+3}\right] .
$$

Proof We consider the function $\bar{G}(x, \bar{\xi}):=G(x, \pi-\bar{\xi})$ with $\bar{\xi} \in\left(0, \frac{\pi}{N+1}\right)$. For $N \geqslant 3$, odd integer, we have that

$$
\begin{aligned}
\bar{G}(x, \bar{\xi}) & =\frac{1}{2 \pi} \sum_{m=1}^{\infty}(-1)^{m+1} \frac{\phi_{m}}{m^{3}} \sin (m x) \sin (m \bar{\xi}) \\
& =\frac{1}{2 \pi} \sum_{m=1}^{N}\left[\frac{\phi_{m}}{m^{3}} \sin (m x) \sin (m \bar{\xi})-\frac{\phi_{m+1}}{(m+1)^{3}} \sin [(m+1) x] \sin [(m+1) \bar{\xi}]\right] \\
& +\frac{1}{2 \pi} \sum_{m=N+2}^{\infty d d}(-1)^{m+1} \frac{\phi_{m}}{m^{3}} \sin (m x) \sin (m \bar{\xi}) \quad \forall x, \bar{\xi} \in\left(0, \frac{\pi}{N+1}\right) .
\end{aligned}
$$

By Theorem 2.1, we know that $\phi_{m}>0$ and is strictly decreasing with respect to $m \in \mathbb{N}^{+}$for all $y, \eta \in[-\ell, \ell]$; hence, we have

$$
\begin{aligned}
\phi_{1} \sin (x) \sin (\bar{\xi}) & -\frac{\phi_{2}}{2^{3}} \sin (2 x) \sin (2 \bar{\xi})=\sin (x) \sin (\bar{\xi})\left[\phi_{1}-\frac{\phi_{2}}{2} \cos (x) \cos (\bar{\xi})\right] \\
& >\frac{\phi_{2}}{2} \sin (x) \sin (\bar{\xi})>\frac{\phi_{N+1}}{2} \sin (x) \sin (\bar{\xi}) \quad \forall x, \bar{\xi} \in\left(0, \frac{\pi}{2}\right), \quad \forall N \geqslant 3 .
\end{aligned}
$$

Exploiting the inequality

$$
\begin{aligned}
& \frac{\sin (m x) \sin (m \bar{\xi})}{m^{3}}-\frac{\sin [(m+1) x] \sin [(m+1) \bar{\xi}]}{(m+1)^{3}}>\sin (x) \sin (\bar{\xi})\left[\frac{1}{m^{\frac{3}{2}}}-\frac{1}{(m+1)^{\frac{3}{2}}}\right]^{2} \\
& \forall x, \bar{\xi} \in\left(0, \frac{\pi}{N+1}\right), \forall m=3, \ldots, N, \quad \forall N \geqslant 3, \text { odd },
\end{aligned}
$$

(see Lemma 6.4 in "Appendix" for a proof) and (58), we get

$$
\begin{aligned}
& \sum_{\substack{m=1 \\
\text { odd }}}^{N}\left[\frac{\phi_{m}}{m^{3}} \sin (m x) \sin (m \bar{\xi})-\frac{\phi_{m+1}}{(m+1)^{3}} \sin [(m+1) x] \sin [(m+1) \bar{\xi}]\right] \\
& >\phi_{N+1} \sin (x) \sin (\bar{\xi})\left[\frac{1}{2}+\sum_{\substack{m=3 \\
\text { odd }}}^{N}\left(\frac{1}{m^{\frac{3}{2}}}-\frac{1}{(m+1)^{\frac{3}{2}}}\right)^{2}\right] \quad \forall x, \bar{\xi} \in\left(0, \frac{\pi}{N+1}\right) \forall N \geqslant 3, \text { odd. }
\end{aligned}
$$


On the other hand, since $|\sin (m \bar{\xi})|<m \sin (\bar{\xi})$ for all $\bar{\xi} \in(0, \pi)$ and through the monotonicity of the $\phi_{m}$, we get

$$
\left|\sum_{m=N+2}^{\infty}(-1)^{m+1} \frac{\phi_{m}}{m^{3}} \sin (m x) \sin (m \bar{\xi})\right| \leqslant \phi_{N+1} \sin (\bar{\xi}) \sum_{m=N+2}^{\infty} \frac{1}{m^{2}} \quad \forall \bar{\xi} \in(0, \pi), \forall N \geqslant 3 .
$$

From (59)-(60), for all $N \geqslant 3$ odd, we infer

$$
\bar{G}(x, \bar{\xi}) \geqslant \frac{\phi_{N+1}}{2 \pi} \sin (\bar{\xi})\left[\frac{1}{2}+\sum_{\substack{m=3 \\ \text { odd }}}^{N}\left(\frac{1}{m^{\frac{3}{2}}}-\frac{1}{(m+1)^{\frac{3}{2}}}\right)^{2}\right]\left(\sin (x)-\bar{C}_{N}\right) \quad \forall x, \bar{\xi} \in\left(0, \frac{\pi}{N+1}\right),
$$

where

$$
\bar{C}_{N}:=\frac{\sum_{m=N+2}^{\infty} \frac{1}{m^{2}}}{\frac{1}{2}+\sum_{\substack{m=3 \\ \text { odd }}}^{N}\left[\frac{1}{m^{\frac{3}{2}}}-\frac{1}{(m+1)^{\frac{3}{2}}}\right]^{2}} .
$$

Next, we denote by $\bar{x}_{N}$ the unique solution to the equation

$$
\sin \left(\bar{x}_{N}\right)=\bar{C}_{N} \quad \bar{x}_{N} \in(0, \pi / 2) .
$$

The above definition makes sense for all $N \geqslant 3$, odd, since the map $N \mapsto \bar{C}_{N}$ is positive, strictly decreasing and $0<\bar{C}_{N}<1$. We prove that

$$
\bar{C}_{N}<\sin \left(\frac{\pi}{N+3}\right) \quad \forall N \geqslant 3 \text {, odd. }
$$

To this aim, we note that

$$
\frac{1}{2}+\sum_{\substack{m=3 \\ \text { odd }}}^{N}\left[\frac{1}{m^{\frac{3}{2}}}-\frac{1}{(m+1)^{\frac{3}{2}}}\right]^{2}>\frac{1}{2} \text { and } \sum_{m=N+2}^{\infty} \frac{1}{m^{2}} \leqslant \int_{N+1}^{\infty} \frac{1}{x^{2}} d x=\frac{1}{N+1}
$$

implying

$$
\bar{C}_{N}<\frac{2}{N+1} \quad \forall N \geqslant 3
$$

From (56), (62) follows by noticing that $\frac{2}{N+1} \leqslant \frac{3}{N+3} \leqslant \sin \left(\frac{\pi}{N+3}\right)$ for all $N \geqslant 3$. Recalling that $G(x, \xi)=\bar{G}(x, \pi-\xi)$, the above estimate yields the proof of the first part of the statement of Lemma 5.4. Similarly, the proof of the second part follows by exploiting the fact that $\bar{G}(x, \bar{\xi})=\bar{G}(\bar{\xi}, x)$ for all $(x, \bar{\xi}) \in[0, \pi] \times[0, \pi]$, by which

$$
\bar{G}(x, \bar{\xi})>0 \quad \forall(x, \bar{\xi}) \in\left(0, \frac{\pi}{N+1}\right) \times\left[\frac{\pi}{N+3}, \frac{\pi}{N+1}\right) .
$$


Finally, by Lemma 5.4, we obtain:

Proposition 5.5 There holds

$$
G(x, \xi)>0 \quad \forall(x, \xi) \in\left(0, \frac{\pi}{4}\right) \times\left(\frac{3}{4} \pi, \pi\right) \quad \text { and } \quad \forall(x, \xi) \in\left(\frac{3}{4} \pi, \pi\right) \times\left(0, \frac{\pi}{4}\right) .
$$

Proof Fixing $N \geqslant 3$, odd integer, from Lemma 5.4 we have that

$$
G(x, \xi)>0 \quad \forall(x, \xi) \in\left(0, \frac{\pi}{N+1}\right) \times\left(\pi-\frac{\pi}{N+1}, \pi\right) \backslash\left(0, \frac{\pi}{N+3}\right) \times\left(\pi-\frac{\pi}{N+3}, \pi\right),
$$

and taking the infinite union of the above sets (over $N \geqslant 3$ odd), we conclude that

$$
G(x, \xi)>0 \quad \forall(x, \xi) \in\left(0, \frac{\pi}{4}\right) \times\left(\frac{3}{4} \pi, \pi\right),
$$

which is the first part of the statement of Proposition 5.5. The second part simply follows by (47).

Proof of Theorem 2.2 completed The proof follows by combining the statements of Propositions 5.1, 5.3 and 5.5.

Acknowledgements The authors are members of the Gruppo Nazionale per l'Analisi Matematica, la Probabilità e le loro Applicazioni (GNAMPA) of the Istituto Nazionale di Alta Matematica (INdAM) and are partially supported by the INDAM-GNAMPA 2019 grant: "Analisi spettrale per operatori ellittici con condizioni di Steklov o parzialmente incernierate" and by the PRIN project 201758MTR2: "Direct and inverse problems for partial differential equations: theoretical aspects and applications" (Italy).

Funding Open access funding provided by Politecnico di Torino within the CRUI-CARE Agreement.

Open Access This article is licensed under a Creative Commons Attribution 4.0 International License, which permits use, sharing, adaptation, distribution and reproduction in any medium or format, as long as you give appropriate credit to the original author(s) and the source, provide a link to the Creative Commons licence, and indicate if changes were made. The images or other third party material in this article are included in the article's Creative Commons licence, unless indicated otherwise in a credit line to the material. If material is not included in the article's Creative Commons licence and your intended use is not permitted by statutory regulation or exceeds the permitted use, you will need to obtain permission directly from the copyright holder. To view a copy of this licence, visit http://creativecommons.org/licenses/by/4.0/.

\section{Appendix 1}

In this section, we prove an inequality used in Sect. 4 to get the monotonicity issue (6) of Theorem 2.1.

Lemma 6.1 Given $F(z)$ and $\bar{F}(z)$ as in $(10)$, there holds: 


$$
\frac{2 F(z)-F^{\prime}(z)}{[F(z)]^{2}} \pm\left[\frac{2 \bar{F}(z)-\bar{F}^{\prime}(z)}{[\bar{F}(z)]^{2}}\right]<0 \quad \forall z>0
$$

Proof We have

$$
\begin{aligned}
& 2 F(z)-F^{\prime}(z)=(3+\sigma)[\sinh (2 z)-\cosh (2 z)]+(1-\sigma)(1-2 z):=\alpha(z) \\
& 2 \bar{F}(z)-\bar{F}^{\prime}(z)=(3+\sigma)[\sinh (2 z)-\cosh (2 z)]-(1-\sigma)(1-2 z)
\end{aligned}
$$

and we observe that $\alpha(0)=-2(1+\sigma)<0$ and $\alpha(z) \sim-2 z(1-\sigma) \rightarrow-\infty$ for $z \rightarrow+\infty$; $\alpha(z)$ has stationary points satisfying $(3+\sigma)[\sinh (2 \bar{z})-\cosh (2 \bar{z})]=-(1-\sigma)$, hence $\alpha(\bar{z})=-2(1-\sigma) \bar{z}<0$ for all $\bar{z}>0$, implying $\alpha(z)<0$ for all $z>0$. From above and recalling that $\bar{F}(z)>F(z)>0$ for all $z>0$, a sufficient condition for the thesis is: $2 F(z)-F^{\prime}(z) \pm\left[2 \bar{F}(z)-\bar{F}^{\prime}(z)\right]<0$ for all $z>0$. To this aim, we notice that:

$$
\begin{aligned}
& 2 F(z)-F^{\prime}(z)+2 \bar{F}(z)-\bar{F}^{\prime}(z)=2(3+\sigma)[\sinh (2 z)-\cosh (2 z)]<0 \quad \forall z>0 \\
& 2 F(z)-F^{\prime}(z)-2 \bar{F}(z)+\bar{F}^{\prime}(z)=2(1-\sigma)(1-2 z)<0 \quad \forall z>\frac{1}{2} .
\end{aligned}
$$

Therefore, it remains to prove the negativity of the term below for all $0<z \leqslant 1 / 2$ :

$$
\begin{aligned}
& \frac{2 F(z)-F^{\prime}(z)}{[F(z)]^{2}}-\frac{2 \bar{F}(z)-\bar{F}^{\prime}(z)}{[\bar{F}(z)]^{2}} \\
& =\frac{(1-\sigma)(3+\sigma)^{2}}{8[F(z) \bar{F}(z)]^{2}}\left\{e^{4 z}(1-2 z)+e^{-4 z}(1+6 z)-4 z-2+16 \frac{(1-\sigma)^{2}}{(3+\sigma)^{2}}\left(z^{2}-2 z^{3}\right)\right\} \\
& \leqslant \frac{(1-\sigma)(3+\sigma)^{2}}{8[F(z) \bar{F}(z)]^{2}}\left\{e^{4 z}(1-2 z)+e^{-4 z}(1+6 z)-4 z-2+\frac{16}{9}\left(z^{2}-2 z^{3}\right)\right\} \\
& :=\frac{(1-\sigma)(3+\sigma)^{2}}{8[F(z) \bar{F}(z)]^{2}} \rho(z) .
\end{aligned}
$$

We have $\rho(0)=\rho^{\prime}(0)=0$ and $\frac{32}{9} \rho^{\prime \prime}(z)=9\left[-z e^{4 z}+e^{-4 z}(3 z-1)\right]+1-6 z:=\bar{\rho}(z)$. For $z \in\left[\frac{1}{6}, \frac{1}{2}\right]$ we get $\bar{\rho}(z) \leqslant 9 z\left[e^{-4 z}-e^{4 z}\right]<0$, while for $z \in\left(0, \frac{1}{6}\right]$ we have $\bar{\rho}(z) \leqslant 1-\frac{9}{2} e^{-\frac{2}{3}}<0$, implying $\rho(z)<0$ for all $z \in\left(0, \frac{1}{2}\right]$ and, in turn, the thesis.

\section{Appendix 2}

In this section, we prove two inequalities that we repeatedly exploit in the estimates of the Fourier series of the Green function in Sect. 5.

Lemma 6.2 Let $N \geqslant 2$ be an integer. There holds

$$
\sin (m x) \sin (m \xi)>\sin (x) \sin (\xi) \quad \forall x, \xi \in\left(0, \frac{\pi}{N+1}\right), \quad \forall m=2, \ldots, N
$$

Proof Let $m \geqslant 2$ be an integer. It is readily seen that 


$$
\sin (m x)-\sin (x)=0 \quad \Longleftrightarrow \quad x=\frac{2 k \pi}{m-1} \vee x=\frac{(2 k+1) \pi}{m+1} \quad \text { for } \quad k \in \mathbb{Z}
$$

By this, since $(\sin (m x)-\sin (x))^{\prime}(0)=m-1>0$, we infer that $\sin (m x)-\sin (x)>0$ for all $x \in\left(0, \frac{\pi}{m+1}\right)$ and, in turn, the thesis.

Before stating and proving the second inequality, we need the following lemma.

Lemma 6.3 Let $m \geqslant 3$ be an integer and set $a_{m}:=\left[\frac{1}{m^{3 / 2}}-\frac{1}{(m+1)^{3 / 2}}\right]^{2}$. The function

$$
v_{m}(t):=\frac{\sin (m t)}{m^{2}}-\frac{\sin [(m+1) t]}{(m+1)^{2}}-a_{m} \sin (t), \quad t \in\left[-\frac{2 \pi}{m+1}, \frac{2 \pi}{m+1}\right]
$$

vanishes at $t=0$ and $t= \pm t_{1}$ with $t_{1} \in\left(\frac{2 \pi}{2 m+1}, \frac{3 \pi}{2 m}\right)$. Furthermore, $v_{m}(t)>0$ in $\left[-\frac{2 \pi}{m+1},-t_{1}\right)$ and $\left(0, t_{1}\right)$ while $v_{m}(t)<0$ in $\left(-t_{1}, 0\right)$ and $\left(t_{1}, \frac{2 \pi}{m+1}\right]$.

Proof of Theorem 2.2 completed Since $v_{m}$ is odd, it is sufficient to study its behavior in $\left[0, \frac{2 \pi}{m+1}\right]$. We compute

$$
\begin{aligned}
v_{m}^{\prime}(t) & =\frac{\cos (m t)}{m}-\frac{\cos [(m+1) t]}{m+1}-a_{m} \cos (t), \\
v_{m}^{\prime \prime}(t) & =\sin [(m+1) t]-\sin (m t)+a_{m} \sin (t) .
\end{aligned}
$$

For all $m \geqslant 3$, we have that $v_{m}^{\prime}(0)=\frac{m^{2}-m-1}{m^{3}(m+1)}+\frac{1}{(m+1)^{3 / 2}}\left(\frac{2}{m^{3 / 2}}-\frac{1}{(m+1)^{3 / 2}}\right)>0$ and, by exploiting the fact that $\sin \left(\frac{2(m+1) \pi}{2 m+1}\right)=-\sin \left(\frac{2 m \pi}{2 m+1}\right)=-\sin \left(\frac{\pi}{2 m+1}\right)$ and (56), that $v_{m}\left(\frac{2 \pi}{2 m+1}\right)>\sin \left(\frac{\pi}{2 m+1}\right)\left[\frac{1}{m^{2}}+\frac{1}{(m+1)^{2}}\right]-\frac{1}{m^{3}}>0$. Let us write

$$
v_{m}^{\prime \prime}(t)=2\left[\cos \frac{(2 m+1) t}{2}+a_{m} \cos \frac{t}{2}\right] \sin \frac{t}{2}=: 2 w(t) \sin \frac{t}{2} .
$$

Clearly, $w^{\prime}<0$ in $\left[0, \frac{2 \pi}{2 m+1}\right]$; since $w(0)>0$ and $w\left(\frac{2 \pi}{2 m+1}\right)<0$, we infer that $v_{m}^{\prime \prime}$ is first positive and then negative in $\left[0, \frac{2 \pi}{2 m+1}\right]$. Since $v_{m}(0)=0, v_{m}^{\prime}(0)>0$ and $v_{m}\left(\frac{2 \pi}{2 m+1}\right)>0$, we conclude that $v_{m}>0$ in $\left[0, \frac{2 \pi}{2 m+1}\right]$.

Now, by exploiting the complex identities for the trigonometric functions, we observe that

$$
\begin{array}{llll}
\sin [(m+1) \bar{t}]-\sin (m \bar{t})=0 & \Longleftrightarrow \quad \bar{t}=2 k \pi, \frac{(1+2 k) \pi}{2 m+1} \quad \forall k \in \mathbb{Z} \\
\cos [(m+1) \bar{t}]-\cos (m \bar{t})=0 & \Longleftrightarrow \quad \bar{t}=2 k \pi, \frac{2 k \pi}{2 m+1} \quad \forall k \in \mathbb{Z} .
\end{array}
$$

Hence, $\quad \sin [(m+1) t]>\sin (m t)$ for $t \in\left(0, \frac{\pi}{2 m+1}\right) \cup\left(\frac{3 \pi}{2 m+1}, \frac{2 \pi}{m+1}\right]$ and $v_{m}^{\prime \prime}(t)>0$ for $t \in\left(0, \frac{\pi}{2 m+1}\right) \cup\left(\frac{3 \pi}{2 m+1}, \frac{2 \pi}{m+1}\right]$. Since $v_{m}\left(\frac{3 \pi}{2 m}\right)=-\frac{1}{m^{2}}-\frac{1}{(m+1)^{2}} \sin \left[(m+1) \frac{3 \pi}{2 m}\right]-a_{m} \sin \left(\frac{3 \pi}{2 m}\right)<0$ and $v_{m}\left(\frac{2 \pi}{m+1}\right)<0$, the convexity of $v_{m}(t)$ implies that $v_{m}(t)<0$ for $t \in\left[\frac{3 \pi}{2 m}, \frac{2 \pi}{m+1}\right]$.

On the other hand, from the fact that $\cos (m t)<\cos [(m+1) t]$ for $t \in\left(\frac{2 \pi}{2 m+1}, \frac{2 \pi}{m+1}\right]$ and $\cos (m t)<0$ for $t \in\left(\frac{2 \pi}{2 m+1}, \frac{3 \pi}{2 m}\right)$, we get $\cos (m t)<\frac{m}{m+1} \cos [(m+1) t]$ for $t \in\left(\frac{2 \pi}{2 m+1}, \frac{3 \pi}{2 m}\right)$. This 
implies $v_{m}^{\prime}(t)<0$ for $t \in\left(\frac{2 \pi}{2 m+1}, \frac{3 \pi}{2 m}\right)$ and yields the existence of a unique zero for $v_{m}(t)$ in $\left(\frac{2 \pi}{2 m+1}, \frac{3 \pi}{2 m}\right)$ which is the thesis.

Lemma 6.4 Let $N \geqslant 3$ be an integer. For all $x, \xi \in\left(0, \frac{\pi}{N+1}\right)$ and all $m=3, \ldots, N$, there holds

$$
\frac{\sin (m x) \sin (m \xi)}{m^{3}}-\frac{\sin [(m+1) x] \sin [(m+1) \xi]}{(m+1)^{3}}>\sin (x) \sin (\xi)\left[\frac{1}{m^{\frac{3}{2}}}-\frac{1}{(m+1)^{\frac{3}{2}}}\right]^{2} .
$$

Proof As in the previous lemma, we set $a_{m}:=\left[\frac{1}{m^{3 / 2}}-\frac{1}{(m+1)^{3 / 2}}\right]^{2}$ and we consider the function

$$
\bar{S}_{m}(x, \xi)=\frac{\sin (m x) \sin (m \xi)}{m^{3}}-\frac{\sin [(m+1) x] \sin [(m+1) \xi]}{(m+1)^{3}}-a_{m} \sin (x) \sin (\xi)
$$

with $Q:=\left[0, \frac{\pi}{m+1}\right]^{2}$. By Weierstrass Theorem, $\bar{S}_{m}$ admits maximum and minimum in $Q$. To locate the stationary points of $\bar{S}_{m}$, it is convenient to exploit the change of variables: $(l, \gamma):=(\xi-x, \xi+x)$ according to which $\bar{S}_{m}$ reads

$$
\begin{aligned}
\bar{S}_{m}(l, \gamma)=\frac{1}{2} & \left(\frac{\cos (m \imath)-\cos (m \gamma)}{m^{3}}-\frac{\cos [(m+1) l]-\cos [(m+1) \gamma]}{(m+1)^{3}}\right. \\
& \left.-a_{m}[\cos (l)-\cos (\gamma)]\right)
\end{aligned}
$$

for $(l, \gamma) \in Q_{1}:=\left[-\frac{\pi}{m+1}, \frac{\pi}{m+1}\right] \times\left[0, \frac{2 \pi}{m+1}\right]$. For $v_{m}$ as defined in the statement of Lemma 6.3 , we have

$$
\left\{\begin{array}{l}
\frac{\partial \bar{S}_{m}}{\partial l}(l, \gamma)=-\frac{v_{m}(l)}{2}=0 \\
\frac{\partial \bar{S}_{m}}{\partial \gamma}(l, \gamma)=\frac{v_{m}(\gamma)}{2}=0 .
\end{array}\right.
$$

By Lemma 6.3 , it is readily deduced that $\bar{S}_{m}$ admits only two stationary points $(0,0)$ and $\left(0, t_{1}\right)$ with $t_{1} \in\left(\frac{2 \pi}{2 m+1}, \frac{3 \pi}{2 m}\right)$. Since $(0,0) \in \partial Q_{1}$, we only need to study the nature of $\left(0, t_{1}\right)$. We have $\frac{\partial^{2} \bar{S}_{m}\left(0, t_{1}\right)}{\partial t^{2}}=-v_{m}^{\prime}(0)<0, \frac{\partial^{2} \bar{S}_{m}}{\partial t \partial \gamma}\left(0, t_{1}\right)=0$ and $\frac{\partial^{2} \bar{S}_{m}\left(0, t_{1}\right)}{\partial \gamma^{2}}=v_{m}^{\prime}\left(t_{1}\right)<0$, see the proof of Lemma 6.3. This implies that $\left(0, t_{1}\right)$ is a maximum point. Coming back to the original variables, from the above analysis we infer that $\left(\frac{t_{1}}{2}, \frac{t_{1}}{2}\right)$ is a local maximum point for $\bar{S}_{m}$. About $\bar{S}_{m}(x, \xi)$ constrained to $\partial Q$, we have: $\bar{S}_{m}(x, 0)=\bar{S}_{m}(0, \xi)=0$ for all $x, \xi \in\left[0, \frac{\pi}{m+1}\right]$; hence, we only need to study $\bar{S}_{m}\left(x, \frac{\pi}{m+1}\right)$ and $\bar{S}_{m}\left(\frac{\pi}{m+1}, \xi\right)$ for all $x, \xi \in\left[0, \frac{\pi}{m+1}\right]$; since they have the same analytic expression, we only focus on

$$
\begin{aligned}
\bar{S}_{m}\left(x, \frac{\pi}{m+1}\right)= & \sin \left(\frac{\pi}{m+1}\right)\left[\frac{\sin (m x)}{m^{3}}-a_{m} \sin (x)\right]:=\sin \left(\frac{\pi}{m+1}\right) \tilde{w}_{m}(x) \\
& \text { for } x \in\left[0, \frac{\pi}{m+1}\right],
\end{aligned}
$$


where $\sin \left(\frac{m \pi}{m+1}\right)=\sin \left(\frac{\pi}{m+1}\right)>0$ for all $m \geqslant 3$. We have $\widetilde{w}_{m}(0)=0$ and $\widetilde{w}_{m}\left(\frac{\pi}{m+1}\right)=\sin \left(\frac{\pi}{m+1}\right) \frac{1}{(m+1)^{\frac{3}{2}}}\left[\frac{2}{m^{\frac{3}{2}}}-\frac{1}{(m+1)^{\frac{3}{2}}}\right]>0$ for all $m \geqslant 3$. Moreover, since $\sin (m x)-\sin (x)>0$ for all $x \in\left(0, \frac{\pi}{m+1}\right)$ and $\frac{1}{m}>a_{m}$ for all $m \in \mathbb{N}^{+}$, we get $\widetilde{w}_{m}^{\prime \prime}(x)=-\left[\frac{\sin (m x)}{m}-a_{\underline{m}} \sin (x)\right]<0$ for $x \stackrel{m+1}{\in}\left[0, \frac{\pi}{m+1}\right]$, implying $\widetilde{w}_{m}(x) \geqslant 0$ for all $x \in\left[0, \frac{\pi}{m+1}\right]$. Once established that $\bar{S}_{m}(x, \xi)$ is nonnegative on $\partial Q$ and admits no internal minimum points in $Q$, we conclude that $\bar{S}_{\underline{m}}(x, \xi) \geqslant 0$ for all $(x, \xi) \in Q$. Finally, the strict inequality in (64) comes by observing that $S_{m}(x, \xi)=0$ for $(x, \xi) \in Q$, if and only if $x=0$ or $\xi=0$.

\section{References}

1. Berchio, E., Buoso, D., Gazzola, F., Zucco, D.: A Minimaxmax Problem for Improving the Torsional Stability of Rectangular Plates. J. Optim. Theory Appl. 177, 64-92 (2018)

2. Berchio, E., Falocchi, A.:Maximizing the ratio of eigenvalues of non-homogeneous partially hinged plates. J. Spect. Theory (to appear)

3. Berchio, E., Falocchi, A., Ferrero, A., Ganguly, D.:On the first frequency of reinforced partially hinged plates, Commun. Contemp. Math. (2019)

4. Berchio, E., Ferrero, A., Gazzola, F.: Structural instability of nonlinear plates modelling suspension bridges: mathematical answers to some long-standing questions. Nonlin. Anal. Real World Appl. 28, 91-125 (2016)

5. Boggio, T.: Sull'equilibrio delle piastre elastiche incastrate. Rend. Acc. Lincei 10, 197-205 (1901)

6. Boggio, T.: Sulle funzioni di Green d'ordine m. Rend. Circ. Mat. Palermo 20, 97-135 (1905)

7. Coffman, C.V., Duffin, R.J.: On the structure of biharmonic functions satisfying the clamped plate conditions on a right angle. Adv. Appl. Math. 1, 373-389 (1980)

8. Ferrero, A., Gazzola, F.: A partially hinged rectangular plate as a model for suspension bridges. Disc. Cont. Dyn. Syst. A. 35, 5879-5908 (2015)

9. Gazzola, F., Grunau, H.C., Sweers, G.: Polyharmonic boundary value problems, LNM 1991 Springer (2010)

10. Grunau, H.C., Romani, G.:Unexpected differences between fundamental solutions of general higherorder elliptic operators and of products of second-order operators, arXiv: 1902.06503

11. Grunau, H.C., Sweers, G.: A clamped plate with a uniform weight may change sign. Discrete Contin. Dyn. Syst. Ser. S7(4), 761-766 (2014)

12. Grunau, H.C., Sweers, G.: In any dimension a "clamped plate" with a uniform weight may change sign. Nonlinear Anal. 97, 119-124 (2014)

13. Hardy, G.H., Rogosinski, W.W.: Fourier Series. Cambridge University Press, Cambridge (1956)

14. Mansfield, E.H.: The bending and stretching of plates, 2nd edn. Cambridge University Press, Cambridge (2005)

15. Nazarov, S., Sweers, G.: A hinged plate equation and iterated Dirichlet Laplace operator on domains with concave corners. J. Differ. Equ. 233(1), 151-180 (2007)

16. Parini, E., Stylianou, A.: On the positivity preserving property of hinged plates. SIAM J. Math. Anal. 41(5), 2031-2037 (2009)

17. Romani, G.: Positivity for fourth-order semilinear problems related to the Kirchhoff-Love functional. Anal. PDE 10(4), 943-982 (2017)

18. Schnieders, I., Sweers, G.: A biharmonic converse to Krein-Rutman: a maximum principle near a positive eigenfunction. Positivity 24, 677 (2019)

19. Sweers, G.: On sign preservation for clotheslines, curtain rods, elastic membranes and thin plates. Jahresber. Dtsch. Math.-Ver. 118(4), 275-320 (2016)

20. Sweers, G.: When is the first eigenfunction for the clamped plate equation of fixed sign? Electron. J. Differ. Equ. Conf. 6, 285-296 (2001)

21. Sweers, G., Vassi, K.: Positivity for a hinged convex plate with stress. SIAM J. Math. Anal. 50(1), 1163-1174 (2018)

Publisher's Note Springer Nature remains neutral with regard to jurisdictional claims in published maps and institutional affiliations. 